\title{
Analysis and Design of Digital IIR Integrators and Differentiators Using Minimax and Pole, Zero, and Constant Optimization Methods
}

\author{
Madhu Jain, ${ }^{1}$ Maneesha Gupta, ${ }^{2}$ and N. K. Jain ${ }^{3}$ \\ ${ }^{1}$ Department of Electronics and Communication Engineering, Jaypee Institute of Information Technology, A-10, Sector 62, \\ Noida, Uttar Pradesh 201307, India \\ ${ }^{2}$ Advanced Electronics Lab, Division of Electronics and Communication Engineering, Netaji Subhas Institute of Technology, \\ Sector 3, Dwarka, New Delhi 110075, India \\ ${ }^{3}$ Instrument Design and Development Centre, Indian Institute of Technology, Hauz Khas, New Delhi 110016, India
}

Correspondence should be addressed to Madhu Jain; ermadhu2003@gmail.com

Received 17 April 2013; Accepted 7 May 2013

Academic Editors: C. W. Chiou, E. I. El-Masry, and E. Tlelo-Cuautle

Copyright (c) 2013 Madhu Jain et al. This is an open access article distributed under the Creative Commons Attribution License, which permits unrestricted use, distribution, and reproduction in any medium, provided the original work is properly cited.

Proposed work deals with the design of a family of stable IIR digital integrators via use of minimax and pole, zero, and constant optimization methods. First the minimax optimization method is used to design a family of second-, third-, and fourth-order digital integrators by optimizing the magnitude response in a min-max sense under the satisfactory condition of constant group delay. Then the magnitude and group delay response is further improved using pole, zero, and constant optimization method. Subsequently, by modifying the transfer function of all of the designed integrators appropriately, new differentiators are obtained. Simulation results show that proposed approach outperforms existing design methods in terms of both magnitude and phase response.

\section{Introduction}

Digital integrators and differentiators are integral parts of many systems like digital signal processing, control, audio, and video processing, communication, and medical applications. Frequency response of an ideal digital integrator is $1 / j \omega$ and of ideal digital differentiator is $j \omega$, where $j=\sqrt{ }-1$ and $\omega$ is the angular frequency in radians/second. Due to increasing number of applications involving digital signal processing, variety of requirements that have to be met by digital integrators and differentiators have increased as well. Digital integrators and differentiators can be classified as finite impulse response (FIR) and infinite impulse response (IIR), respectively. For a large number of applications, where high selectivity and efficient processing of digital signal are required, IIR digital integrators and differentiators are preferred.

Generally IIR digital integrators and differentiators designs have been proposed by using the Newton-Cotes integration rule [1-13]. These digital operators can be designed directly or by transformation of analog integrators and differentiators like impulse invariance, bilinear transformation, forward difference equation, and backward difference equation [1-3]. Rectangular, Trapezoidal, Simpson 1/3, Simpson $3 / 8$, and Boole are the basic integrators proposed [1-3]. Schneider et al. [4] have used parabolic and cubic numerical integration rules in design of digital integrator. Al-Alaoui [5] has designed digital integrator by using linear interpolation method (mixing of trapezoidal and rectangular integrators); since then, this method has gained immense popularity. Ngo [6] has designed an integrator by applying the $z$-transform on one of the closed-form Newton-Cotes integration rule. Tseng and Lee [7] have used fractional delay in design of digital integrator. Gupta et al. [8-13] have also used interpolation of various existing integrators to design digital integrators.

Optimization [14-23] is a very popular design method. It is widely used to improve the performance of a system by reducing its runtime, bandwidth, memory requirement, or any other property. Optimization methods such as linear, simulated annealing, genetic algorithm, and pole-zero optimization have been used earlier to design IIR digital 
integrators and differentiators [14-19]. Papamarkos and Chamzas [14] have used linear programming optimization method to design digital integrators. Al-Alaoui [15] has also proposed a family of digital integrators by using interpolation and simulated annealing optimization method. Upadhyay and Singh [16] have used pole-zero optimization method in design of digital integrators. Jiang and Kwan [17] have used semidefinite programming (SDP) relaxation algorithm to design IIR digital filters in the minimax sense. Lai and Lin [18] have used a sequential constrained least-squares method to design IIR digital filters in the minimax sense. Hsu et al. [19] have used genetic algorithm optimization method in the design of digital integrators.

Al-Alaoui $[15,24]$ has proposed a method to design IIR digital differentiators, based on the inversion and magnitude stabilization of the transfer function of IIR digital integrators. This technique also became very popular and was used by various researchers like Bihan [1], Ngo [6], Gupta et al. [8-10], and Upadhyay and Singh [16] in the design of digital differentiators. The accuracy and range in terms of percentage absolute relative error (PARE) of the magnitude response of the designed differentiators are the same as those of integrators.

In this proposed work, a family of stable IIR digital integrators is designed via the use of minimax $[17,18]$ and pole, zero and constant (PZC) optimization methods. The combination of two optimization techniques in a design is previously used by Renders and Flasse and Wu et al. [20, 21]. Renders and Flasse [20] have used genetic algorithm with hill-climbing optimization in order to generate a global optimization algorithm and Wu et al. [21] have used particle swarm with cultural algorithm to enhance the convergence characteristics of the original particle swarm optimization.

Here, minimax optimization method is used to design a family of second-, third-, and fourth-order digital integrators by optimizing the magnitude response in a min-max sense under the satisfactory condition of constant group delay. Then the PZC optimization is applied on minimax-optimized integrators to further improve frequency (magnitude and phase) response. This design strategy is motivated by the fact that there is a probability that minimax optimization does not provide global optimum solution so the PZC optimization is used over the result of minimax optimization to greatly increase the probability of finding the true global optimum solution. Thereafter, by modifying the transfer function of designed integrators appropriately, new second-, third-, and fourth-order digital differentiators are obtained which have the same accuracy as the designed integrators. These designed wideband operators (integrators and differentiators) outperform all the existing wideband operators in both magnitude and phase response.

All the figures in this paper have been obtained by using MATLAB 7. This paper is organized as follows. Section 2 presents the problem formulation. Section 3 presents the solution methodology consisting of minimax and pole, zero, and constant (PZC) optimization. Section 4 contains performance results by minimax and PZC optimization and comparison of the designed integrators with existing integrators. Section 5 presents the proposed differentiators and their comparison with the existing differentiators. The conclusions are given in Section 6.

\section{Problem Formulation}

It is known that IIR integrators have much better magnitude response than FIR integrators of the same order but their phase characteristics are not linear which can cause problems in some of the signal processing applications. In direct design method for IIR integrators, the desired digital transfer function is obtained directly from the given magnitude, with or without a specified phase specification. Let $I(z)$ be the transfer function of an IIR digital integrator with real coefficients $\left(a_{n}, b_{m}\right)$; then

$$
I(z)=\frac{A(z)}{B(z)}=\frac{\sum_{n=0}^{N} a_{n} z^{-n}}{1+\sum_{m=1}^{N} b_{m} z^{-m}},
$$

where $N$ is the order of the integrator. $I(z)$ can also be defined as

$$
I(z)=\frac{a^{T} f_{N}(z)}{1+b^{T} f_{M}(z)}
$$

where $a=\left[a_{0}, a_{1}, \ldots, a_{N}\right]^{T}, b=\left[b_{1}, b_{2}, \ldots, b_{N}\right]^{T}, f_{N}(z)=$ $\left[1, z^{-1}, z^{-2}, \ldots, z^{-N}\right]^{T}$, and $f_{M}(z)=\left[z^{-1}, z^{-2}, \ldots, z^{-N}\right]^{T}$, the superscript $T$ is used to define transpose.

The frequency response $I\left(e^{j \omega}\right)$ of the integrator can be obtained from its transfer function $I(z)$ by simply evaluating it on the unit circle; that is,

$$
I\left(e^{j \omega}\right)=\left.I(z)\right|_{z=e^{j \omega}}=\left|I\left(e^{j \omega}\right)\right| e^{j \theta(\omega)} .
$$

Here, $\left|I\left(e^{j \omega}\right)\right|$ is the amplitude and $\theta(\omega)$ is the phase of the digital integrator, respectively.

Group delay is a measure of phase distortion; it is calculated by differentiating phase with respect to frequency. The degree of nonlinearity of the phase indicates the deviation of the group delay from a constant.

The group delay of integrator $I\left(e^{j \omega}\right)$ is defined as

$$
\tau_{g}(\omega)=-\frac{d \theta(\omega)}{d \omega}
$$

The main limitation in the design of IIR digital integrators is to meet the specified magnitude and phase characteristics. An integrator should have linear phase response over the wideband frequency range; otherwise it would introduce phase distortion. Various researchers have been trying to improve the performance of digital integrators but a common difficulty faced by them is that when the magnitude response is improved, the phase response gets distorted and vice versa.

\section{Solution Methodologies}

In this paper, a family of second-, third-, and fourth-order stable wideband digital integrators are designed via use of minimax and pole, zero, and constant optimization. The flow chart of complete design method is shown in Figure 1. 


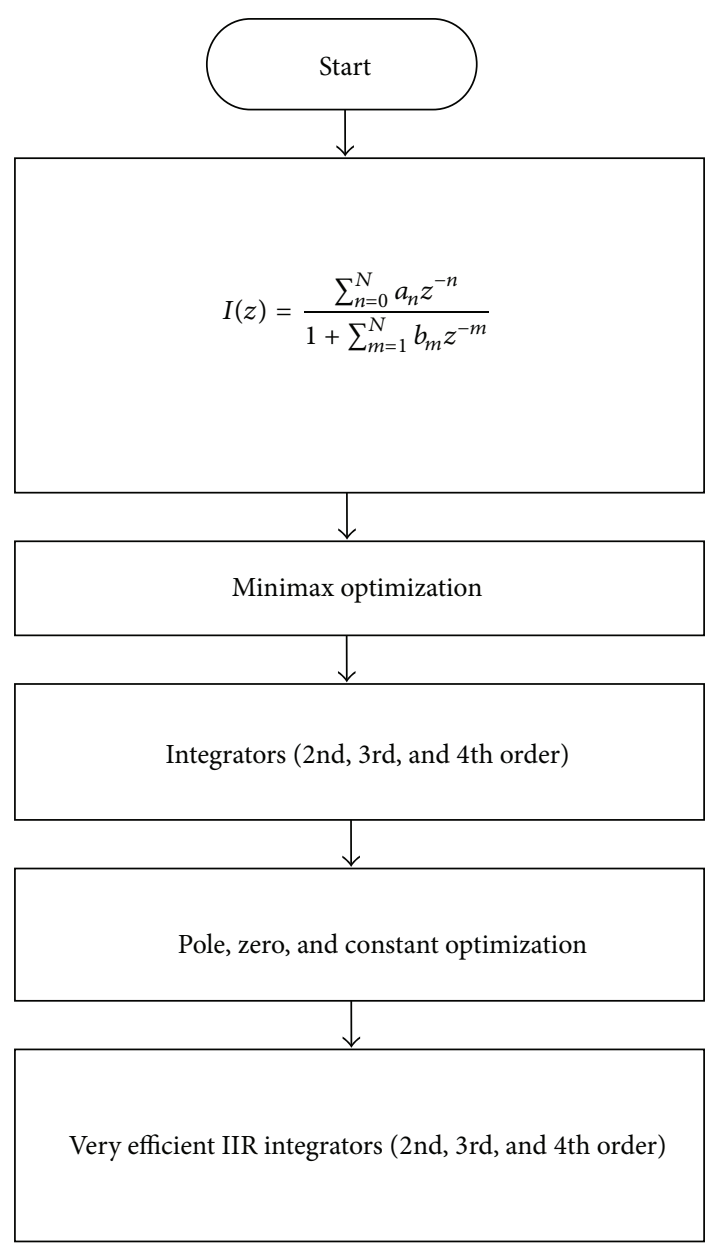

FIGURE 1: Flowchart of the complete design method for digital integrator.

\subsection{Design of IIR Digital Integrators Using Minimax Optimiza-} tion. A minimax is one of the optimization techniques in which the task is to find the solution with the minimum of the worst (maximum) value. It has been popularly used to find the unknown system parameters by minimizing the maximum difference between system output and design specifications $[17,18]$. The flow chart of the applied minimax optimization for second-order digital integrator is shown in Figure 2.

The transfer function of an integrator with unknown coefficients is defined as $I\left(e^{j \omega}\right)$. A vector containing numerator and denominator coefficients is defined as $x=\left[a^{T}, b^{T}\right]^{T}$. In order to explicitly show the dependence of the frequency response of integrator $I\left(e^{j \omega}\right)$ on the numerator and denominator coefficients, the integrator is defined as $I\left(e^{j \omega}, x\right)$, in place of $I\left(e^{j \omega}\right)$. Here, $M(\omega, x)$ and $\mathrm{M}_{\text {int }}(\omega)$ are the amplitude of the designed and ideal integrator, respectively. Percentage absolute relative error (PARE) for magnitude response is defined as an objective function and order of the filter is defined by the value of $N$. Here coefficients $a_{n}$ and $b_{m}$ are taken as optimization parameters:

$$
\operatorname{PARE}(\omega, x)=\frac{\left|M_{\mathrm{int}}(\omega)-M(\omega, x)\right|}{M_{\mathrm{int}}(\omega)} * 100 .
$$

The applied minimax optimization method can be defined as

$$
\begin{array}{ll}
\operatorname{minimize} & \mu \\
\text { subject to } & |\operatorname{PARE}(\omega, x)| \leq \mu .
\end{array}
$$

In order to obtain exact approximation of the magnitude response, the PARE values should be as small as possible. For the case of second-order integrator $(N=2)$, the minimum $\left(l_{b}\right)$, maximum $\left(u_{b}\right)$, and initial value $\left(x_{0}\right)$ of coefficients are defined as follows

$$
\begin{aligned}
& l_{b}=\left[\begin{array}{lllll}
-0.9999 & -0.9999 & -0.9999 & -0.9999 & -0.9999
\end{array}\right], \\
& u_{b}=\left[\begin{array}{lllll}
+0.9999 & +0.9999 & +0.9999 & +0.9999 & +0.9999
\end{array}\right], \\
& x_{0}=\left[\begin{array}{lllll}
+0.0010 & +0.0010+0.0010 & +0.0010 & +0.0010
\end{array}\right] .
\end{aligned}
$$

The lengths of the coefficients are taken as 1 integer and 4 decimal points. The same algorithm is also applied for 1 integer 3 decimal and 1 integer 5 decimal length but the difference in the efficiency is negligible.

The constraint of linear phase is applied by restricting the maximum value of absolute group delay $\left(\tau_{g}(\omega, x)\right)$ of integrator $I\left(e^{j \omega}, x\right)$ to be less than 5 samples. The frequency range is defined as $0 \leq \omega \leq \pi$ radians/second.

The internal function of minimax optimization in MATLAB 7 (fminimax) is used to obtain the coefficients for minimum of maximum $\operatorname{PARE}(\omega, x)$ over the defined frequency range.

The following command is used for this purpose:

$$
\begin{array}{r}
{[x, \text { PAREval, maxPARE }]} \\
=\text { fminimax }\left(@ \operatorname{PARE}(\omega, x), x_{0},\right. \\
{[],[],[],[], l_{b}, u_{b},} \\
\left.@ \tau_{g}(\omega, x)<5\right),
\end{array}
$$

where $x$ is the vector of coefficients having minimum of maximum PARE for the defined frequency range.

PAREval and maxPARE are the vectors of PARE values over the defined frequency range and maximum among these corresponding to coefficients $x$.

Four blank square brackets are used to define that there are no linear equalities and inequalities.

fminimax function works in following manner.

In first iteration, a set of coefficients are selected; then the values of objective function (PARE) are calculated by using (5), corresponding to these coefficients. The selection criteria of the coefficients consist of two points: first the value of coefficient should be within defined minimum and maximum bound, and the absolute value of group delay of the corresponding integrator should be less than 5 samples. In next iteration, another set of coefficients are selected and PARE values are calculated. This process is repeated till suitable coefficients are obtained. After last iteration, maximum PARE of each iteration is compared and coefficients corresponding 


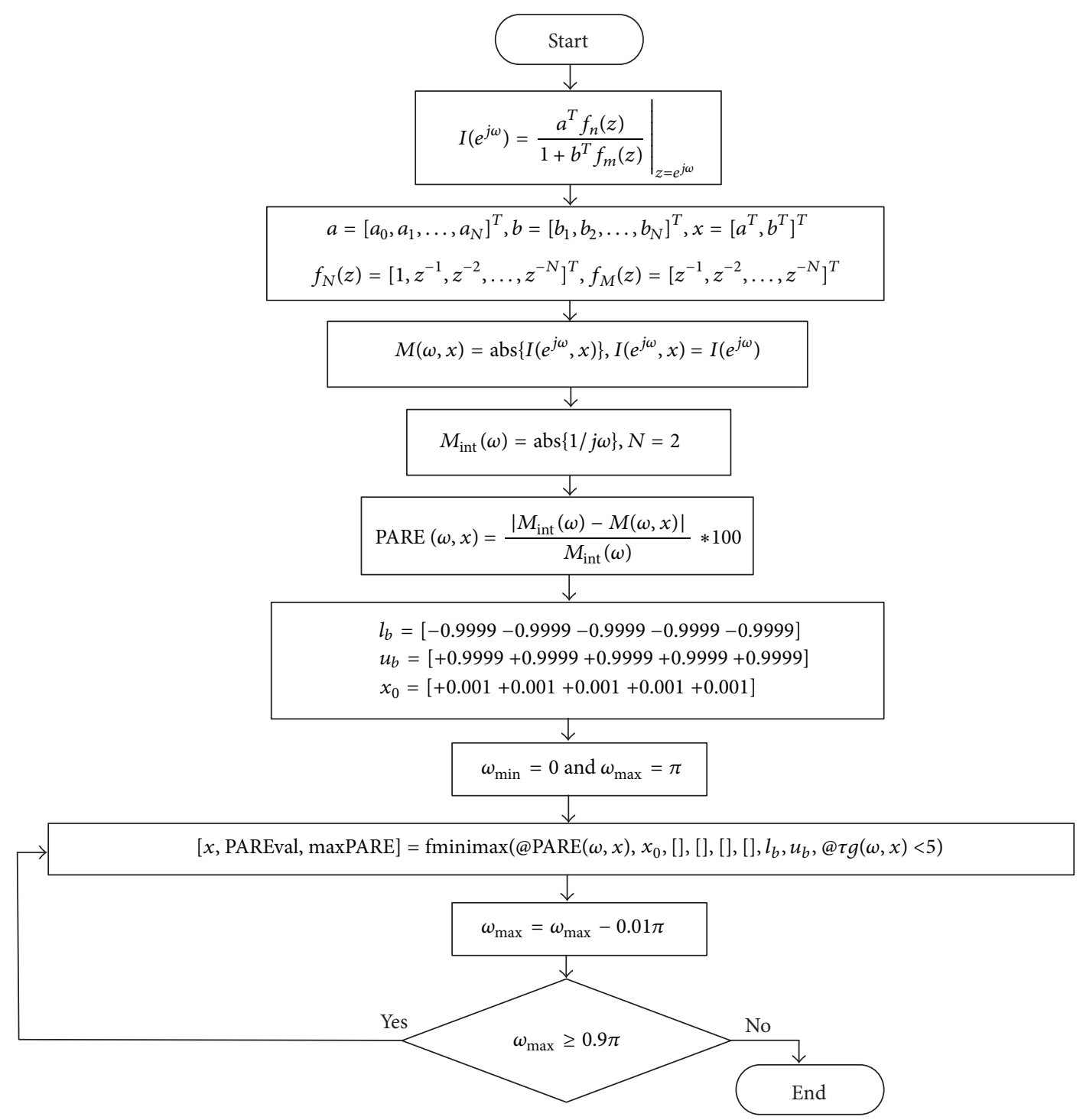

FIGURE 2: Flowchart of the applied minimax optimization in design of second-order digital integrator.

to minimum among maximum PARE are the desired coefficients.

After this, frequency range is defined as $0 \leq \omega \leq$ $0.99 \pi$ radians/second, by reducing maximum frequency by $0.01 \pi$ radians/second and again coefficients are obtained for minimum of maximum PAREs using the command defined in (7). This process is repeated eleven times $(0 \leq \omega \leq \pi$ radians/second to $0 \leq \omega \leq 0.90 \pi$ radians/second), so that designed integrators can be considered as wideband. In the last, all eleven sets of coefficients are listed in a table along with mean and maximum PARE of the corresponding integrator. By comparison, the optimum coefficients are the one having minimum among maximum PAREs. By substituting these coefficients in (2), a second-order digital integrator is obtained having magnitude response close to the ideal one and almost linear phase response.

The same algorithm is used to design third- and fourthorder digital integrators. In order to improve the optimization result, PZC optimization method is applied on the result of minimax optimization.

3.2. Pole, Zero, and Constant (PZC) Optimization Method. The location of poles and zeros of the transfer function is very important for digital system analyses and synthesis. According to the location of poles, it is possible to test stability of the system. Variation of poles and zeros has a significant effect on the response of a design. The available literature [16, 22,23 ] shows that pole-zero optimization is popular among researchers to improve their responses in various fields. The basic general strategy of designing frequency selective filters is also based on pole-zero placements. As placing a pole near the frequency $\omega_{0}$ on the unit circle will increase the gain of the frequency response near $\omega_{0}$, similarly, a zero near $\omega_{0}$ will diminish the gain near $\omega_{0}$. Here, as all the three parameters, namely, poles, zeros, and constant, are optimized, the results show tremendous improvement as compared to 
the previously proposed operators, where only poles and zeros have been optimized.

To obtain the poles, zeros, and constant of an integrator $I(z)$, the numerator and denominator polynomials of (1) are rewritten as

$$
I(z)=\frac{a_{0}+a_{1} z^{-1}+a_{2} z^{-2}+\cdots+a_{N} z^{-N}}{1+b_{1} z^{-1}+b_{2} z^{-2}+\cdots+b_{N} z^{-N}}
$$

or

$$
\begin{gathered}
I(z)=\left(a _ { 0 } z ^ { - N } \left(z^{N}+\left(a_{1} / a_{0}\right) z^{N-1}\right.\right. \\
\left.\left.+\left(a_{2} / a_{0}\right) z^{N-2}+\cdots+\left(a_{N} / a_{0}\right)\right)\right) \\
\times\left(z ^ { - N } \left(z^{N}+b_{1} z^{N-1}+b_{2} z^{N-2}\right.\right. \\
\left.\left.+\cdots+b_{N}\right)\right)^{-1} .
\end{gathered}
$$

The numerator and denominator can be factorized and expressed as

$$
\begin{gathered}
I(z)=a_{0} * \frac{\left(z-z_{1}\right)\left(z-z_{2}\right) \cdots\left(z-z_{N}\right)}{\left(z-p_{1}\right)\left(z-p_{2}\right) \cdots\left(z-p_{N}\right)}, \\
I(z)=k * \frac{\prod_{n=1}^{N}\left(z-z_{n}\right)}{\prod_{n=1}^{N}\left(z-p_{n}\right)},
\end{gathered}
$$

where $k=a_{0}$ is a scaling constant, $z_{n}$ are the zeros, and $p_{n}$ are the poles of integrator $I(z)$, respectively.

For PZC optimization, the modified transfer function $I^{\prime}(z)$ can be written as

$$
\begin{gathered}
I^{\prime}(z)=\frac{(k+\Delta k) \prod_{n=1}^{N}\left(z-z_{n}+\Delta z_{n}\right)}{\prod_{n=1}^{N}\left(z-p_{n}+\Delta p_{n}\right)}, \\
I^{\prime}\left(e^{j \omega}\right)=\left.I^{\prime}(z)\right|_{z=e^{j \omega}} .
\end{gathered}
$$

The amplitude of the modified digital integrator $I^{\prime}\left(e^{j \omega}\right)$ is

$$
\left|I^{\prime}\left(e^{j \omega}\right)\right|=(k+\Delta k) * \frac{\prod_{n=1}^{N}\left|\left(e^{j \omega}-z_{n}+\Delta z_{n}\right)\right|}{\prod_{n=1}^{N}\left|\left(e^{j \omega}-p_{n}+\Delta p_{n}\right)\right|} .
$$

PARE of integrator $I^{\prime}\left(e^{j \omega}\right)$ is defined as

$$
\operatorname{PARE}(\omega)=\frac{\left|M_{\mathrm{int}}(\omega)-\right| I^{\prime}\left(e^{j \omega}\right)||}{M_{\mathrm{int}}(\omega)} * 100
$$

Phase response of the modified digital integrator is defined as

$$
\theta_{I}^{\prime}(\omega)=\sum_{n=1}^{N} \angle\left(e^{j \omega}-z_{n}+\Delta z_{n}\right)-\sum_{n=1}^{N} \angle\left(e^{j \omega}-p_{n}+\Delta p_{n}\right)
$$

Equations (12)-(14) show that the optimization parameters $\Delta_{k}, \Delta z_{n}$, and $\Delta p_{n}$ affect not only magnitude but phase response also.
The group delay of the modified integrator $I^{\prime}\left(e^{j \omega}\right)$ is defined as

$$
\tau_{g}^{\prime}(\omega)=-\frac{d \theta_{I}^{\prime}(\omega)}{d \omega}
$$

Various steps used in the design of PZC optimized second-order IIR digital integrator are as follows.

(i) First of all, minimax optimized second-order integrator is defined in terms of poles, zeros, and constant.

(ii) Percentage absolute relative error $\operatorname{PARE}(\omega)$ as defined in (13) is specified as an objective function.

(iii) Then the lower and upper bound, for all optimization parameters $\left(\Delta_{k}, \Delta z_{n}\right.$, and $\left.\Delta p_{n}\right)$ are defined as $[-0.0010+0.0010]$, respectively.

(iv) The step size is taken as +0.0001 .

(v) All the optimization parameters are varied for 21 values, and those which gives minimum PARE are defined as optimum parameters.

(vi) The above mentioned process is repeated for thirdand fourth-order minimax optimized integrators.

In this way PZC optimized second-, third-, and fourth-order wideband digital integrators are obtained.

\section{Results after Minimax and Pole, Zero, and Constant Optimization Methods}

4.1. Results after Minimax Optimization and Their Comparison with Existing Integrators. The second-, third-, and fourth-order wideband digital integrators are designed for the frequency range of $0 \leq \omega \leq \pi$ radians/second to $0 \leq \omega \leq$ $0.90 \pi$ radians/second. Coefficients and maximum PARE for each frequency range for second-, third-, and fourth-order digital integrators are shown in Tables 1, 2, and 3, respectively.

By observing Tables 1, 2, and 3, the minimum of maximum PARE are obtained for the optimum coefficients as mentioned below.

Second order: $a_{0}=0.8650, a_{1}=0.5993, a_{2}=0.0539$, $b_{1}=-0.4834$, and $b_{2}=-0.5145$.

Third order: $a_{0}=0.8639, a_{1}=0.9997, a_{2}=0.2471$, $a_{3}=0.0058, b_{1}=-0.0220, b_{1}=-0.8315$, and $b_{3}=$ -0.1442 .

Fourth order: $a_{0}=0.8659, a_{1}=0.5426, a_{2}=0.5925$, $a_{3}=0.3480, a_{4}=0.0276, b_{1}=-0.5479, b_{2}=0.1845$, $b_{3}=-0.3429$, and $b_{4}=-0.2905$. 
TABLE 1: Coefficients and maximum PARE for different frequency ranges of second-order integrator.

\begin{tabular}{lccccccc}
\hline S. no. & Frequency range (radians/second) & $a_{0}$ & $a_{1}$ & $a_{2}$ & $b_{1}$ & $b_{2}$ & Maximum PARE \\
\hline 1 & $0.01 \leq \omega \leq \pi$ & +0.8580 & +0.6010 & +0.0545 & -0.4798 & -0.5176 & 1.80 \\
2 & $0.02 \leq \omega \leq 0.99 \pi$ & +0.8599 & +0.6030 & +0.0582 & -0.4772 & -0.5100 & 1.50 \\
3 & $0 \leq \omega \leq 0.98 \pi$ & +0.8631 & +0.5897 & +0.0530 & -0.4982 & -0.5034 & 1.00 \\
4 & $0 \leq \omega \leq 0.97 \pi$ & +0.8657 & +0.5918 & +0.0534 & -0.4975 & -0.5025 & 0.70 \\
$\mathbf{5}$ & $\mathbf{0 . 0 1} \leq \boldsymbol{\omega} \leq \mathbf{0 . 9 6} \pi$ & $+\mathbf{0 . 8 6 5 0}$ & $+\mathbf{0 . 5 9 9 3}$ & $+\mathbf{0 . 0 5 3 9}$ & $-\mathbf{0 . 4 8 3 4}$ & $-\mathbf{0 . 5 1 4 5}$ & $\mathbf{0 . 3 7}$ \\
6 & $0.01 \leq \omega \leq 0.95 \pi$ & +0.8640 & +0.5900 & +0.0544 & -0.4940 & -0.5017 & 0.45 \\
7 & $0.01 \leq \omega \leq 0.94 \pi$ & +0.8660 & +0.5920 & +0.0526 & -0.4890 & -0.5051 & 0.50 \\
8 & $0.02 \leq \omega \leq 0.93 \pi$ & +0.8676 & +0.5937 & +0.0531 & -0.4863 & -0.5034 & 0.75 \\
9 & $0.02 \leq \omega \leq 0.92 \pi$ & +0.8634 & +0.5920 & +0.0519 & -0.4854 & -0.5042 & 0.40 \\
10 & $0.03 \leq \omega \leq 0.91 \pi$ & +0.8615 & +0.5991 & +0.0538 & -0.4736 & -0.5139 & 0.44 \\
11 & $0.03 \leq \omega \leq 0.90 \pi$ & +0.8598 & +0.5981 & +0.0543 & -0.4721 & -0.5121 & 0.51 \\
\hline
\end{tabular}

TABLE 2: Coefficients and maximum PARE for different frequency ranges of third-order integrator.

\begin{tabular}{|c|c|c|c|c|c|c|c|c|c|}
\hline S. no. & Frequency range (radians/second) & $a_{0}$ & $a_{1}$ & $a_{2}$ & $a_{3}$ & $b_{1}$ & $b_{2}$ & $b_{3}$ & Maximum PARE \\
\hline 1 & $0.01 \leq \omega \leq \pi$ & 0.8692 & 0.9978 & 0.2464 & 0.0054 & -0.0252 & -0.8285 & -0.1506 & 1.60 \\
\hline 2 & $0.01 \leq \omega \leq 0.99 \pi$ & 0.8684 & 0.9967 & 0.2475 & 0.0059 & -0.0265 & -0.8274 & -0.1490 & 1.40 \\
\hline 3 & $0.04 \leq \omega \leq 0.98 \pi$ & 0.8598 & 0.9765 & 0.2558 & 0.0078 & -0.0219 & -0.7706 & -0.1592 & 1.10 \\
\hline 4 & $0.05 \leq \omega \leq 0.97 \pi$ & 0.8585 & 0.9760 & 0.2567 & 0.0068 & -0.0237 & -0.7712 & -0.1587 & 0.90 \\
\hline 5 & $0 \leq \omega \leq 0.96 \pi$ & 0.8662 & 0.9987 & 0.2470 & 0.0056 & -0.0239 & -0.8286 & -0.1478 & 0.80 \\
\hline 6 & $0.01 \leq \omega \leq 0.95 \pi$ & 0.8629 & 0.9981 & 0.2519 & 0.0094 & -0.0218 & -0.8287 & -0.1473 & 0.70 \\
\hline 7 & $0.01 \leq \omega \leq 0.94 \pi$ & 0.8626 & 0.9993 & 0.2514 & 0.0083 & -0.0210 & -0.8292 & -0.1467 & 0.45 \\
\hline 8 & $0.01 \leq \omega \leq 0.93 \pi$ & 0.8639 & 0.9997 & 0.2471 & 0.0058 & -0.0220 & -0.8315 & -0.1442 & 0.30 \\
\hline 9 & $0.02 \leq \omega \leq 0.92 \pi$ & 0.8666 & 0.9960 & 0.2503 & 0.0071 & -0.0314 & -0.8223 & -0.1450 & 0.40 \\
\hline 10 & $0.02 \leq \omega \leq 0.91 \pi$ & 0.8676 & 0.9982 & 0.2492 & 0.0061 & -0.0353 & -0.8251 & -0.1486 & 0.42 \\
\hline 11 & $0.02 \leq \omega \leq 0.90 \pi$ & 0.8689 & 0.9909 & 0.2478 & 0.0088 & -0.0495 & -0.8259 & -0.1423 & 0.50 \\
\hline
\end{tabular}

By substituting the above mentioned optimum coefficients in (2), the resulting transfer functions of second-, third, and fourth-order digital integrators $\left(I_{2 \mathrm{MO}}(z), I_{3 \mathrm{MO}}(z)\right.$, and $\left.I_{4 \mathrm{MO}}(z)\right)$ are obtained as

$$
\begin{gathered}
I_{2 \mathrm{MO}}(z)=\frac{\left(0.8650+0.5993 z^{-1}+0.0539 z^{-2}\right)}{\left(1-0.4834 z^{-1}-0.5145 z^{-2}\right)} \\
I_{3 \mathrm{MO}}(z)=\frac{\left(0.8639+0.9997 z^{-1}+0.2471 z^{-2}+0.0058 z^{-3}\right)}{\left(1-0.0220 z^{-1}-0.8315 z^{-2}-0.1442 z^{-3}\right)} \\
I_{4 \mathrm{MO}}(z) \\
=\left(0.8659+0.5426 z^{-1}+0.5925 z^{-2}+0.3480 z^{-3}\right. \\
\left.\quad+0.0276 z^{-4}\right) \\
\times\left(1-0.5479 z^{-1}+0.1845 z^{-2}-0.3429 z^{-3}\right. \\
\left.\quad-0.2905 z^{-4}\right)^{-1} .
\end{gathered}
$$

For comparison, various existing integrators have been considered. These are Ngo integrator $\left(I_{\mathrm{N}}(z)\right)$ [6], Gupta et al. 1 integrator $\left(I_{\mathrm{GJK} 1}(z)\right)[8]$, Gupta et al. 2 integrator
$\left(I_{\mathrm{GJK} 2}(z)\right)$ [9], Gupta et al. 3 integrator $\left(I_{\mathrm{GJK} 3}(z)\right)$ [10], AlAlaoui integrator $\left(I_{\mathrm{A}}(z)\right)[15]$, and Upadhyay and Singh integrator $\left(I_{\mathrm{US}}(z)\right)$ [16]. Their transfer functions are

$$
\begin{aligned}
& I_{\mathrm{N}}(z) \\
& \quad=\frac{T_{S}\left(1+2.3658 z^{-1}\right)\left(1-0.2605 z^{-1}+0.047 z^{-2}\right)}{2.7925\left(1-z^{-1}\right)},
\end{aligned}
$$

$I_{\mathrm{GJK} 1}(z)$

$$
=\frac{0.34 T_{S}\left(1+2.541 z^{-1}\right)\left(1-0.2081 z^{-1}+0.03858 z^{-2}\right)}{\left(1-z^{-1}\right)},
$$

$$
\begin{aligned}
& I_{\mathrm{GJK} 2}(z) \\
& =\frac{0.329 T_{S}\left(1+2.663 z^{-1}\right)\left(1-0.2079 z^{-1}+0.03864 z^{-2}\right)}{\left(1-z^{-1}\right)} \\
& I_{\mathrm{GJK} 3}(z) \\
& =\left(0.38125 T_{S}\left(1+2.285 z^{-1}\right)\right. \\
& \left.\quad \times\left(1-0.247 z^{-1}+0.04543 z^{-2}\right)\right) \\
& \quad \times\left(1-z^{-1}\right)^{-1}
\end{aligned}
$$


TABLE 3: Coefficients and maximum PARE for different frequency ranges of fourth-order integrator.

\begin{tabular}{lccccccccccc}
\hline S. no. & $\begin{array}{c}\text { Frequency range } \\
\text { (radians/second) }\end{array}$ & $a_{0}$ & $a_{1}$ & $a_{2}$ & $a_{3}$ & $a_{4}$ & $b_{1}$ & $b_{2}$ & $b_{3}$ & $b_{4}$ & Maximum PARE \\
\hline 1 & $0.01 \leq \omega \leq \pi$ & 0.8658 & 0.5487 & 0.5904 & 0.3602 & 0.0275 & -0.5434 & 0.1837 & -0.3379 & -0.2912 \\
2 & $0.01 \leq \omega \leq 0.99 \pi$ & 0.8667 & 0.5406 & 0.5912 & 0.3613 & 0.0320 & -0.5598 & 0.1986 & -0.3314 & -0.2945 & 1.70 \\
3 & $0.01 \leq \omega \leq 0.98 \pi$ & 0.8651 & 0.5380 & 0.5901 & 0.3604 & 0.0322 & -0.5479 & 0.1983 & -0.3289 & -0.2954 & 1.20 \\
4 & $0.02 \leq \omega \leq 0.97 \pi$ & 0.8654 & 0.5478 & 0.5925 & 0.3598 & 0.0307 & -0.5439 & 0.1869 & -0.3302 & -0.2959 & 0.90 \\
5 & $0.02 \leq \omega \leq 0.96 \pi$ & 0.8640 & 0.5448 & 0.5917 & 0.3583 & 0.0298 & -0.5432 & 0.1858 & -0.3298 & -0.2962 & 0.70 \\
6 & $0.02 \leq \omega \leq 0.95 \pi$ & 0.8642 & 0.5436 & 0.5898 & 0.3572 & 0.0305 & -0.5435 & 0.1858 & -0.3304 & -0.2975 & 0.60 \\
7 & $0.02 \leq \omega \leq 0.94 \pi$ & 0.8624 & 0.5416 & 0.5886 & 0.3557 & 0.0292 & -0.5429 & 0.1846 & -0.3268 & -0.2981 & 0.49 \\
8 & $0.03 \leq \omega \leq 0.93 \pi$ & 0.8629 & 0.5398 & 0.5891 & 0.3582 & 0.0297 & -0.5432 & 0.1879 & -0.3223 & -0.3021 & 0.42 \\
$\mathbf{9}$ & $\mathbf{0 . 0 1} \leq \boldsymbol{\omega} \leq \mathbf{0 . 9 2 \pi}$ & $\mathbf{0 . 8 6 5 9}$ & $\mathbf{0 . 5 4 2 6}$ & $\mathbf{0 . 5 9 2 5}$ & $\mathbf{0 . 3 4 8 0}$ & $\mathbf{0 . 0 2 7 6}$ & $-\mathbf{0 . 5 4 7 9}$ & $\mathbf{0 . 1 8 4 5}$ & $-\mathbf{0 . 3 4 2 9}$ & $-\mathbf{0 . 2 9 0 5}$ & $\mathbf{0 . 2 0}$ \\
10 & $0.03 \leq \omega \leq 0.91 \pi$ & 0.8636 & 0.5411 & 0.5898 & 0.3558 & 0.0296 & -0.5422 & 0.1853 & -0.3278 & -0.3003 & 0.22 \\
11 & $0.02 \leq \omega \leq 0.90 \pi$ & 0.8655 & 0.5409 & 0.5910 & 0.3473 & 0.0283 & -0.5446 & 0.1858 & -0.3391 & -0.2900 & 0.25 \\
\hline
\end{tabular}

TABLE 4: Comparison between existing and designed integrators.

\begin{tabular}{|c|c|c|c|c|}
\hline S. no. & Integrator & Frequency range (radians/second) & Maximum PARE & Mean PARE \\
\hline 1 & $I_{\mathrm{N}}(\mathrm{z}) \mathrm{Ngo}[6]$ & $0 \leq \omega \leq \pi$ & 6.30 & 2.5613 \\
\hline 2 & $1 I_{\mathrm{GJK} 1}(z)$ Gupta et al. [8] & $0 \leq \omega \leq \pi$ & 3.00 & 0.9756 \\
\hline 3 & $2 I_{\mathrm{GJK} 2}(z)$ Gupta et al. [9] & $0 \leq \omega \leq 0.95 \pi$ & 2.80 & 1.0996 \\
\hline 4 & $3 I_{\mathrm{GJK} 3}(z)$ Gupta et al. [10] & $0 \leq \omega \leq \pi$ & 4.69 & 2.1817 \\
\hline 5 & $I_{\mathrm{A}}(z)$ Al-Alaoui $[15]$ & $0.008 \leq \omega \leq 0.60 \pi$ & 7.0 & 1.8393 \\
\hline 6 & $I_{\mathrm{US}}(z)$ Upadhyay and Singh [16] & $0 \leq \omega \leq 0.94 \pi$ & 0.48 & 0.2658 \\
\hline 7 & $I_{2 \mathrm{MO}}(z)$ & $0.01 \leq \omega \leq 0.96 \pi$ & 0.37 & 0.2184 \\
\hline 8 & $I_{3 \mathrm{MO}}(z)$ & $0.01 \leq \omega \leq 0.93 \pi$ & 0.30 & 0.1337 \\
\hline 9 & $I_{4 \mathrm{MO}}(z)$ & $0.01 \leq \omega \leq 0.92 \pi$ & 0.20 & 0.0980 \\
\hline 10 & $I_{2 \mathrm{MO}+\mathrm{PZC}}(z)$ & $0 \leq \omega \leq 0.95 \pi$ & 0.31 & 0.2011 \\
\hline 11 & $I_{3 \mathrm{MO}+\mathrm{PZC}}(z)$ & $0 \leq \omega \leq 0.94 \pi$ & 0.22 & 0.1272 \\
\hline 12 & $I_{4 \mathrm{MO}+\mathrm{PZC}}(z)$ & $0 \leq \omega \leq 0.91 \pi$ & 0.12 & 0.0720 \\
\hline
\end{tabular}

$$
\begin{gathered}
I_{\mathrm{A}}(z) \\
=\left(T _ { S } \left(0.5678+1.013 z^{-1}+0.5629 z^{-2}\right.\right. \\
\left.\left.+0.5406 z^{-3}+0.1519 z^{-4}\right)\right) \\
\times\left(1-0.2097 z^{-1}-0.2106 z^{-2}\right. \\
\left.\quad-0.114 z^{-3}-0.4636 z^{-4}\right)^{-1}, \\
I_{\mathrm{US}}(z)=\frac{0.8657 T_{S}\left(1+0.681 z^{-1}+0.0628 z^{-2}\right)}{\left(1-0.4975 z^{-1}-0.5025 z^{-2}\right)} .
\end{gathered}
$$

Here the sampling period of the integrator " $T_{S}$ " is assumed as 1 second and the Nyquist frequency is $\pi$ radians/sample. The PARE response of the designed integrators and above mentioned integrators over Nyquist frequency range is shown in Figure 3. The useful frequency range, mean, and maximum PAREs in that frequency range for above mentioned integrators are shown in Table 4. The group delay response of the above mentioned integrators is shown in Figure 4.

It is seen that the maximum deviations from constant group delay in case of $I_{2 \mathrm{MO}}(z), I_{3 \mathrm{MO}}(z)$, and $I_{4 \mathrm{MO}}(z)$ are 0.5 samples, 0.6 samples and 0.4 samples, respectively, while, the maximum deviations from constant group delay in case of existing integrators Ngo [6], Gupta et al. 1 [8], Gupta et al. 2 [9], Gupta et al. 3 [10], Al-Alaoui [15], and Upadhyay and Singh [16] are 1.5 samples, 1.3 samples, 1.2 samples, 1.5 samples, 5.0 samples and 0.7 samples, respectively.

It is verified from Figures 3 and 4 and Table 4 that the minimax optimized designed second-, third-, and fourthorder integrators outperform all the existing integrators over entire Nyquist frequency range.

4.2. Results after PZC Optimization and Their Comparison with the Minimax Optimized Integrators. The frequency response of the minimax optimized digital integrators is then improved by using pole, zero, and constant (PZC) optimization. For this minimax optimized integrators $\left(I_{2 \mathrm{MO}}(z)\right.$, $I_{3 \mathrm{MO}}(z)$, and $\left(I_{4 \mathrm{MO}}(z)\right)(16)$ are defined in terms of pole, zero and constant. As all the coefficients of polynomials in (16) are 


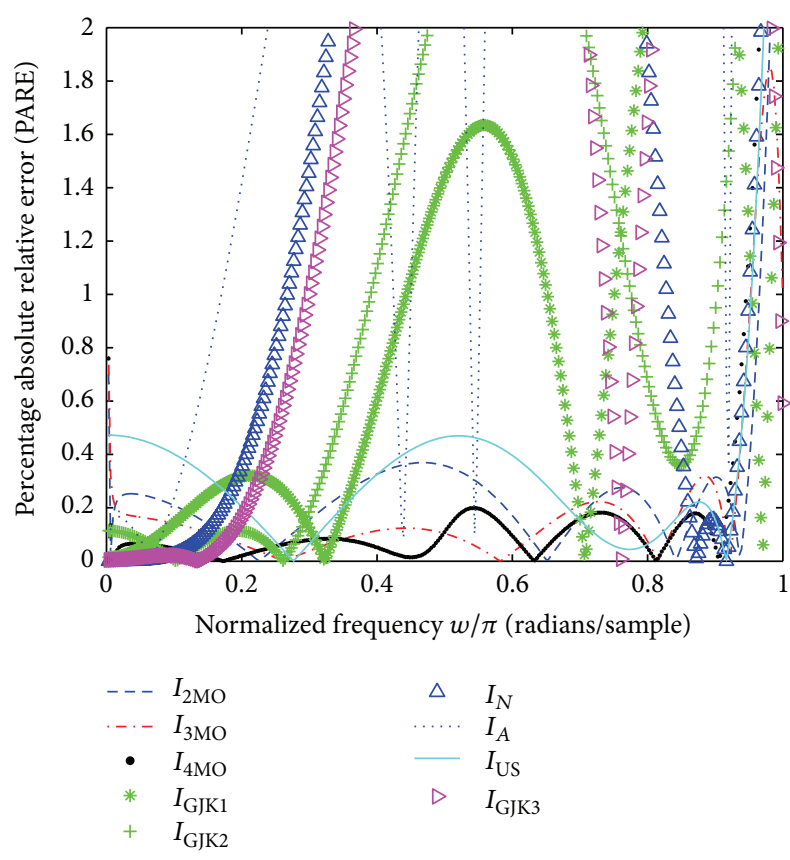

Figure 3: Percentage absolute relative error response of designed integrators; $I_{2 \mathrm{MO}}(z), I_{3 \mathrm{MO}}(z)$, and $I_{4 \mathrm{MO}}(z)$, Ngo integrator $I_{\mathrm{N}}(z)[6]$, Gupta et al. 1 integrator $I_{\mathrm{GJK} 1}(z)$ [8], Gupta et al. 2 integrator $I_{\mathrm{GJK} 2}(z)$ [9], Gupta et al. 3 integrator $I_{\mathrm{GJK} 3}(z)$ [10], Al-Alaoui integrator $I_{\mathrm{A}}(z)$ [15], and Upadhyay and Singh integrator $I_{\mathrm{US}}(z)$ [16].

real, therefore the poles, and zeros will be either real or appear in complex conjugate pairs:

$$
\begin{aligned}
I_{2 \mathrm{MO}}(z)=\frac{0.8650(z+0.1063)(z+0.5865)}{(z-0.9986)(z+0.5152)}, \\
I_{3 \mathrm{MO}}(z)=\frac{0.8639(z+0.8172)(z+0.3139)(z+0.0261)}{(z-0.9989)(z+0.7954)(z+0.1815)}, \\
I_{4 \mathrm{MO}}(z)=(0.8659(z+0.5382)(z+0.0929) \\
\left.\times\left(z-0.7982 e^{j 1.5680}\right)\left(z-0.7982 e^{-j 1.5680}\right)\right) \\
\times\left((z-0.9987)(z+0.4565)\left(z-0.7983 e^{j 1.5672}\right)\right. \\
\left.\times\left(z-0.7983 e^{-j 1.5672}\right)\right)^{-1} .
\end{aligned}
$$

For PZC optimization, the modified transfer function can be written as

$$
\begin{aligned}
I_{2 \mathrm{MO}}^{\prime}(z)= & \left((0.8650+\Delta k)\left(z+0.1063+\Delta z_{1}\right)\right. \\
\times & \left.\left(z+0.5865+\Delta z_{2}\right)\right) \\
\times & \left(\left(z-0.9986+\Delta p_{1}\right)\right. \\
& \left.\times\left(z+0.5152+\Delta p_{2}\right)\right)^{-1}, \\
I_{3 \mathrm{MO}}^{\prime}(z)=( & (0.8639+\Delta k)\left(z+0.8172+\Delta z_{1}\right) \\
\times & \left.\left(z+0.3139+\Delta z_{2}\right)\left(z+0.0261+\Delta z_{3}\right)\right)
\end{aligned}
$$

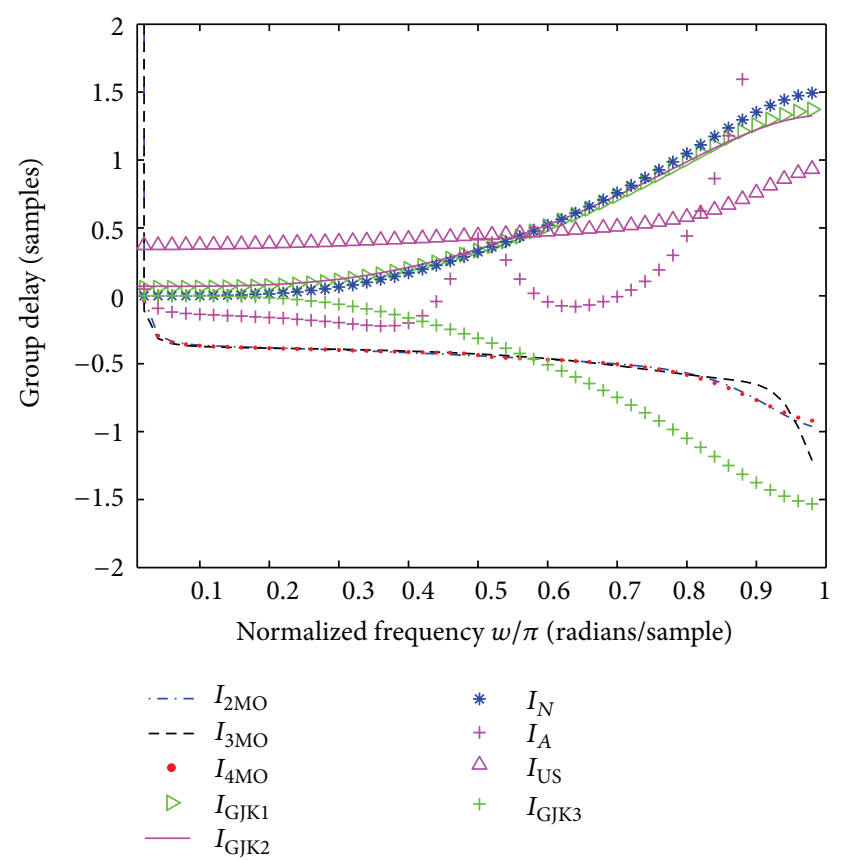

FIGURE 4: Group delay response of designed integrators; $I_{2 \mathrm{MO}}(z)$, $I_{3 \mathrm{MO}}(z)$, and $I_{4 \mathrm{MO}}(z)$, Ngo integrator $I_{\mathrm{N}}(z)$ [6], Gupta et al. 1 integrator $I_{\mathrm{GJK} 1}(\mathrm{z})$ [8], Gupta et al. 2 integrator $I_{\mathrm{GJK} 2}(z)$ [9], Gupta et al. 3 integrator $I_{\mathrm{GJK} 3}(\mathrm{z})$ [10], Al-Alaoui integrator $I_{\mathrm{A}}(z)$ [15], and Upadhyay and Singh integrator $I_{\mathrm{US}}(z)$ [16].

$$
\begin{aligned}
\times & \left(\left(z-0.9989+\Delta p_{1}\right)\left(z+0.7954+\Delta p_{2}\right)\right. \\
& \left.\times\left(z+0.1815+\Delta p_{3}\right)\right)^{-1} \\
I_{4 \mathrm{MO}}^{\prime}(z)=( & (0.8659+\Delta k)\left(z+0.5382+\Delta z_{1}\right) \\
\times & \left(z+0.0929+\Delta z_{2}\right) \\
\times & \left(z-0.7982 e^{j 1.5680}+\Delta z_{3}\right) \\
\times & \left.\left(z-0.7982 e^{-j 1.5680}+\Delta z_{4}\right)\right) \\
\times & \left(\left(z-0.9987+\Delta p_{1}\right)\left(z+0.4565+\Delta p_{2}\right)\right. \\
& \times\left(z-0.7983 e^{j 1.5672}+\Delta p_{3}\right) \\
& \left.\times\left(z-0.7983 e^{-j 1.5672}+\Delta p_{4}\right)\right)^{-1} .
\end{aligned}
$$

After optimization, the optimum values are obtained as follows:

for second order:

$\Delta k=+0.0005, \Delta z_{1}=-0.0003, \Delta z_{2}=+0.0008, \Delta p_{1}=$ -0.0004 , and $\Delta p_{2}=+0.0004$;

for third order:

$\Delta k=+0.0007, \Delta z_{1}=-0.0006, \Delta z_{2}=+0.0009, \Delta z_{3}=$ $-0.0008, \Delta p_{1}=-0.0008, \Delta p_{2}=-0.0007$, and $\Delta p_{3}=$ -0.0008 ; 
for fourth order:

$\Delta k=+0.0004, \Delta z_{1}=-0.0006, \Delta z_{2}=+0.0010$, $\Delta z_{3}=+0.0009 e^{j 1.5680}, \Delta z_{4}=+0.0009 e^{-j 1.5680}, \Delta p_{1}=$ $-0.0008, \Delta p_{2}=+0.0009, \Delta p_{3}=+0.0004 e^{j 1.5672}$, and $\Delta p_{4}=+0.0004 e^{-j 1.5672}$.

By substituting all these values in (19), respectively, the new integrators are obtained as

$$
\begin{aligned}
& I_{2 \mathrm{MO}+\mathrm{PZC}}(z)=\frac{0.8655(z+0.1060)(z+0.5873)}{(z-0.9990)(z+0.5156)}, \\
& I_{3 \mathrm{MO}+\mathrm{PZC}}(z)=(0.8646(z+0.8166)(z+0.3148) \\
&\times(z+0.0253)) \\
& \times((z-0.9997)(z+0.7947) \\
&\quad \times(z+0.1807))^{-1}, \\
& I_{4 \mathrm{MO}+\mathrm{PZC}}(z)=(0.8663(z+0.5376)(z+0.0939) \\
& \times\left(z-0.7973 e^{j 1.5680}\right) \\
&\left.\times\left(z-0.7973 e^{-j 1.5680}\right)\right) \\
& \times((z-0.9995)(z+0.4574) \\
& \times\left(z-0.7979 e^{j 1.5672}\right) \\
&\left.\times\left(z-0.7979 e^{-j 1.5672}\right)\right)^{-1} .
\end{aligned}
$$

All the designed integrators ((18) and (20)) are stable as their respective poles are inside the unit circle. The PARE response of the designed integrators $I_{2 \mathrm{MO}}(z), I_{3 \mathrm{MO}}(z)$, $I_{4 \mathrm{MO}}(z), I_{2 \mathrm{MO}+\mathrm{PZC}}(z), I_{3 \mathrm{MO}+\mathrm{PZC}}(z)$, and $I_{4 \mathrm{MO}+\mathrm{PZC}}(z)$ is shown in Figure 5. The comparison of designed and existing integrators on the basis of mean PARE, maximum PARE and useful frequency range is shown in Table 4.

It is seen that the PZC optimized integrators $I_{2 \mathrm{MO}+\mathrm{PZC}}(z)$, $I_{3 \mathrm{MO}+\mathrm{PZC}}(z)$, and $I_{4 \mathrm{MO}+\mathrm{PZC}}(z)$ have PARE $\leq 0.31$ over $0 \leq \omega \leq 0.95 \pi$ radians/second, $\leq 0.22$ over $0 \leq \omega \leq$ $0.94 \pi$ radians/second, and $\leq 0.12$ over $0 \leq \omega \leq 0.91 \pi$ radians/second, respectively. The group delay response of the designed integrators $I_{2 \mathrm{MO}}(z), I_{3 \mathrm{MO}}(z), I_{4 \mathrm{MO}}(z), I_{2 \mathrm{MO}+\mathrm{PZC}}(z)$, $I_{3 \mathrm{MO}+\mathrm{PZC}}(z)$, and $I_{4 \mathrm{MO}+\mathrm{PZC}}(z)$ is shown in Figure 6 . It is verified from Figures 5 and 6 and Table 4 that PZC optimization improves frequency (both magnitude and phase) response of minimax optimized integrators. In this way, a perfect family of second-, third-, and fourth-order wideband stable digital integrators are designed with superior frequency response.

The square wave response of designed and existing integrators is shown in Figures 7 and 8, respectively. It is seen that the response of designed integrators $I_{2 \mathrm{MO}}(z), I_{3 \mathrm{MO}}(z)$, $I_{4 \mathrm{MO}}(z), I_{2 \mathrm{MO}+\mathrm{PZC}}(z), I_{3 \mathrm{MO}+\mathrm{PZC}}(z)$, and $I_{4 \mathrm{MO}+\mathrm{PZC}}(z)$ is exact triangular wave, while the response of existing integrators is distorted triangular wave.

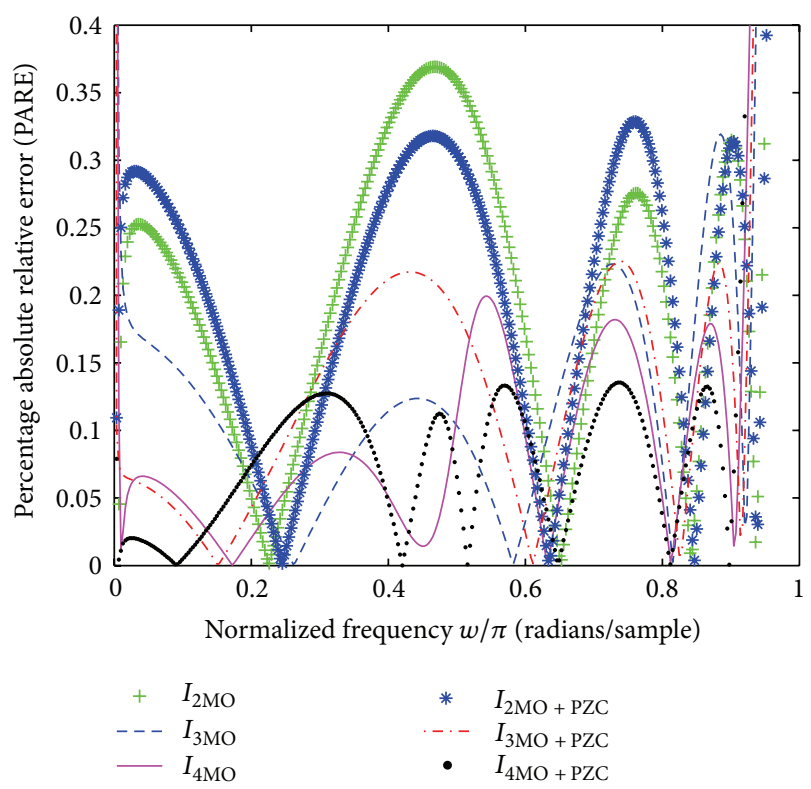

Figure 5: Percentage absolute relative error response of designed integrators; $I_{2 \mathrm{MO}}(z), I_{3 \mathrm{MO}}(z), I_{4 \mathrm{MO}}(z), I_{2 \mathrm{MO}+\mathrm{PZC}}(z), I_{3 \mathrm{MO}+\mathrm{PZC}}(z)$, and $I_{4 \mathrm{MO}+\mathrm{PZC}}(z)$.

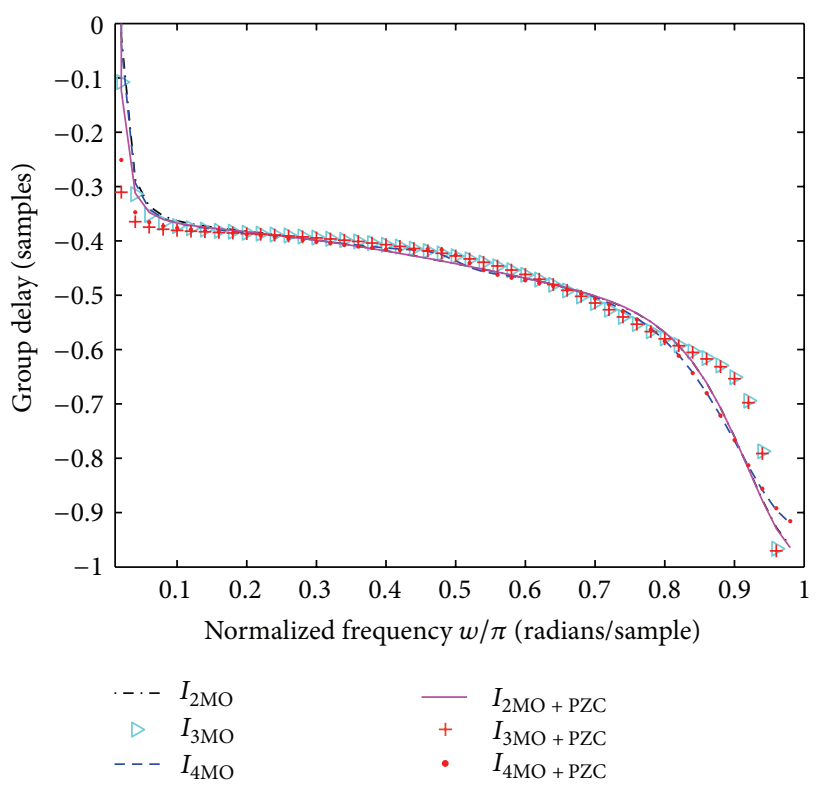

FIGURE 6: Group delay response of designed integrators; $I_{2 \mathrm{MO}}(z)$, $I_{3 \mathrm{MO}}(z), I_{4 \mathrm{MO}}(z), I_{2 \mathrm{MO}+\mathrm{PZC}}(z), I_{3 \mathrm{MO}+\mathrm{PZC}}(z)$, and $I_{4 \mathrm{MO}+\mathrm{PZC}}(z)$.

\section{Design of IIR Digital Differentiators and Their Comparison with the Existing Differentiators}

A digital differentiator can also be designed by using transfer function of digital integrator in a similar way to that used in the design of analog differentiator, as suggested by $\mathrm{Al}$ Alaoui [24]. This method consists of four design steps. In the first step, an integrator is designed that has the same range 


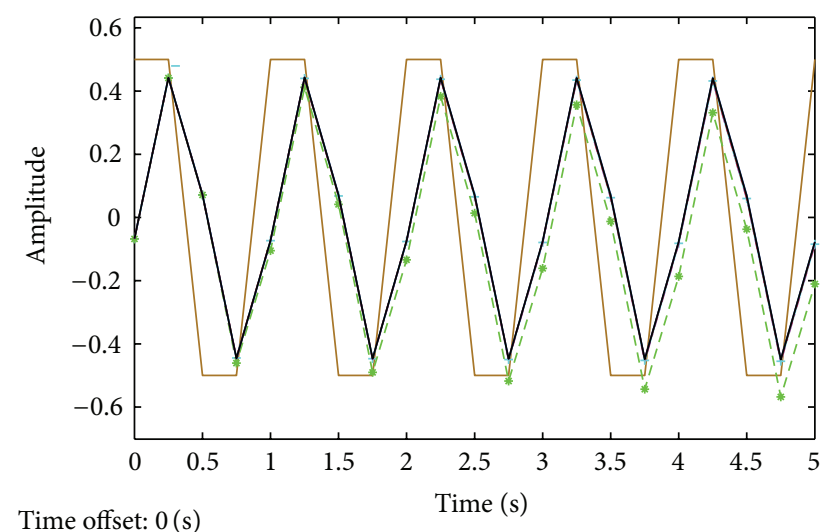

$$
\begin{aligned}
& \text { - Input } \quad--I_{2 \mathrm{MO}}+\mathrm{PZC} \\
& +I_{2 \mathrm{MO}}-I_{3 \mathrm{MO}}+\mathrm{PZC} \\
& \text {-*- } I_{3 \mathrm{MO}} \quad-I_{4 \mathrm{MO}}+\mathrm{PZC} \\
& \cdot-\cdot I_{4 \mathrm{MO}}
\end{aligned}
$$

Figure 7: Square wave response of designed integrators; $I_{2 \mathrm{MO}}(z)$, $I_{3 \mathrm{MO}}(z), I_{4 \mathrm{MO}}(z), I_{2 \mathrm{MO}+\mathrm{PZC}}(z), I_{3 \mathrm{MO}+\mathrm{PZC}}(z)$, and $I_{4 \mathrm{MO}+\mathrm{PZC}}(z)$.

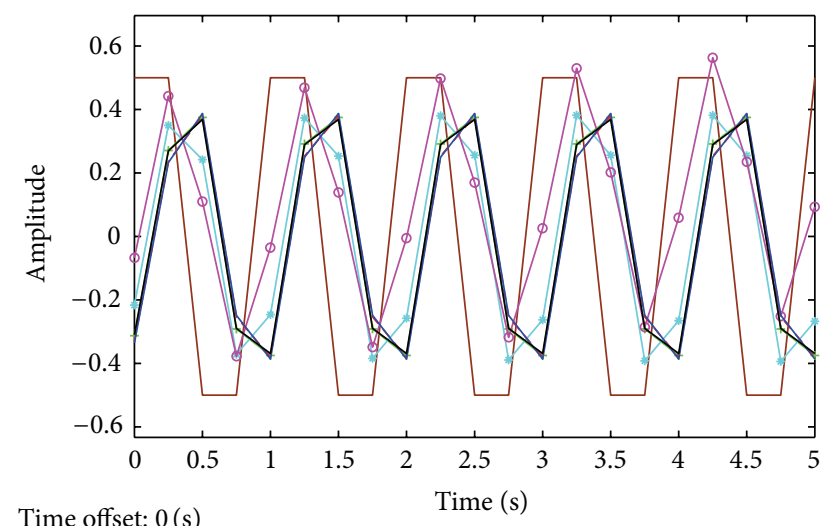

Time offset: $0(\mathrm{~s})$

$$
\begin{array}{ll}
- \text { Input } & --I_{\mathrm{GJK} 1} \\
-I_{N} & -I_{\mathrm{GJK} 2} \\
\rightarrow-I_{A} & -I_{\mathrm{GJK} 3} \\
\rightarrow-I_{\mathrm{US}} &
\end{array}
$$

FIGURE 8: Square wave response of existing integrators; Ngo $I_{\mathrm{N}}(z)$ [6], Gupta et al. $1 I_{\mathrm{GJK} 1}(z)$ [8], Gupta et al. $2 I_{\mathrm{GJK} 2}(z)$ [9], Gupta et al. $3 I_{\mathrm{GJK} 3}(z)$ [10], Al-Alaoui $I_{\mathrm{A}}(z)$ [15], and Upadhyay and Singh integrator $I_{\mathrm{US}}(z)[16]$.

and accuracy as the desired differentiator. Then, the transfer function of this integrator is inverted and stabilized by reflecting the poles that lie outside the unit circle to inside the unit circle, and in the last step, the magnitude is compensated appropriately.

In this section, a family of second-, third-, and fourth-order digital differentiators have been obtained by inverting the transfer functions of designed integrators $I_{2 \mathrm{MO}}(z), I_{3 \mathrm{MO}}(z), I_{4 \mathrm{MO}}(z), I_{2 \mathrm{MO}+\mathrm{PZC}}(z), I_{3 \mathrm{MO}+\mathrm{PZC}}(z)$, and $I_{4 \mathrm{MO}+\mathrm{PZC}}(z)$ defined in (18) and (20), respectively.

The resultant transfer functions are

$$
D_{2 \mathrm{MO}}(z)=\frac{(z-0.9986)(z+0.5152)}{0.8650(z+0.1063)(z+0.5865)},
$$

$$
\begin{aligned}
& D_{3 \mathrm{MO}}(z)=((z-0.9989)(z+0.7954) \\
& \times(z+0.1815)) \\
& \times(0.8639(z+0.8172) \\
& \times(z+0.3139)(z+0.0261))^{-1}, \\
& D_{4 \mathrm{MO}}(\mathrm{z})=((z-0.9987)(z+0.4565) \\
& \times\left(z-0.7983 e^{j 1.5672}\right) \\
& \left.\times\left(z-0.7983 e^{-j 1.5672}\right)\right) \\
& \times((0.8659)(z+0.5382) \\
& \times(z+0.0929) \\
& \times\left(z-0.7982 e^{j 1.5680}\right) \\
& \left.\times\left(z-0.7982 e^{-j 1.5680}\right)\right)^{-1}, \\
& D_{2 \mathrm{MO}+\mathrm{PZC}}(z)=\frac{(z-0.9990)(z+0.5156)}{0.8655(z+0.1060)(z+0.5873)}, \\
& D_{3 \mathrm{MO}+\mathrm{PZC}}(z)=((z-0.9997)(z+0.7947) \\
& \times(z+0.1807)) \\
& \times(0.8646(z+0.8166) \\
& \times(z+0.3148)(z+0.0253))^{-1}, \\
& D_{4 \mathrm{MO}+\mathrm{PZC}}(z)=((z-0.9995)(z+0.4574) \\
& \times\left(z-0.7979 e^{j 1.5672}\right) \\
& \left.\times\left(z-0.7979 e^{-j 1.5672}\right)\right) \\
& \times((0.8663)(z+0.5376) \\
& \times(z+0.0939) \\
& \times\left(z-0.7973 e^{j 1.5680}\right) \\
& \left.\times\left(z-0.7973 e^{-j 1.5680}\right)\right)^{-1} .
\end{aligned}
$$

As all the poles of designed differentiators (see (21)) are inside the unit circle, therefore stabilization and compensation of these transfer functions are not required. To compare the efficiency of these designed differentiators, various recently proposed differentiators have been considered. These are Ngo differentiator $\left(D_{\mathrm{N}}(z)\right)$ [6], Gupta et al. 1 differentiator $\left(D_{\mathrm{GJK} 1}(z)\right)$ [8], Gupta et al. 2 differentiator $\left(D_{\mathrm{GJK} 2}(z)\right)$ [9], Gupta et al. 3 differentiator $\left(D_{\mathrm{GJK} 3}(z)\right)[10]$, Al-Alaoui differentiator $\left(D_{\mathrm{A}}(z)\right)$ [15], and Upadhyay and Singh differentiator $\left(D_{\mathrm{US}}(z)\right)[16]$. 
TABLE 5: Comparison between existing and designed differentiators.

\begin{tabular}{lcccc}
\hline S. no. & Differentiator & Frequency range (radians/second) & Maximum PARE & Mean PARE \\
\hline 1 & $D_{\mathrm{N}}(z)$ Ngo [6] & $0 \leq \omega \leq \pi$ & 4.90 & 2.6997 \\
2 & $1 D_{\mathrm{GJK} 1}(z)$ Gupta et al. [8] & $0 \leq \omega \leq \pi$ & 3.00 & 0.9756 \\
3 & $2 D_{\mathrm{GJK} 2}(z)$ Gupta et al. [9] & $0 \leq \omega \leq 0.95 \pi$ & 2.80 & 4.91 \\
4 & $3 D_{\mathrm{GJK} 3}(z)$ Gupta et al. [10] & $0 \leq \omega \leq \pi$ & 3.80 & 2.1605 \\
5 & $D_{\mathrm{A}}(z)$ Al-Alaoui [15] & $0.04 \leq \omega \leq \pi$ & 0.48 & 1.8362 \\
6 & $D_{\mathrm{US}}(z)$ Upadhyay and Singh [16] & $0 \leq \omega \leq 0.94 \pi$ & 0.37 & 0.2658 \\
7 & $D_{2 \mathrm{MO}}(z)$ & $0.01 \leq \omega \leq 0.96 \pi$ & 0.30 & 0.2184 \\
8 & $D_{3 \mathrm{MO}}(z)$ & $0.01 \leq \omega \leq 0.93 \pi$ & 0.20 & 0.1337 \\
9 & $D_{4 \mathrm{MO}}(z)$ & $0.01 \leq \omega \leq 0.92 \pi$ & 0.31 & 0.0980 \\
10 & $D_{2 \mathrm{MO}+\mathrm{PZC}}(z)$ & $0 \leq \omega \leq 0.95 \pi$ & 0.22 & 0.1271 \\
11 & $D_{3 \mathrm{MO}+\mathrm{PZC}}(z)$ & $0 \leq \omega \leq 0.94 \pi$ & 0.12 & 0.0720 \\
12 & $D_{4 \mathrm{MO}+\mathrm{PZC}}(z)$ & $0 \leq \omega \leq 0.91 \pi$ & & \\
\hline
\end{tabular}

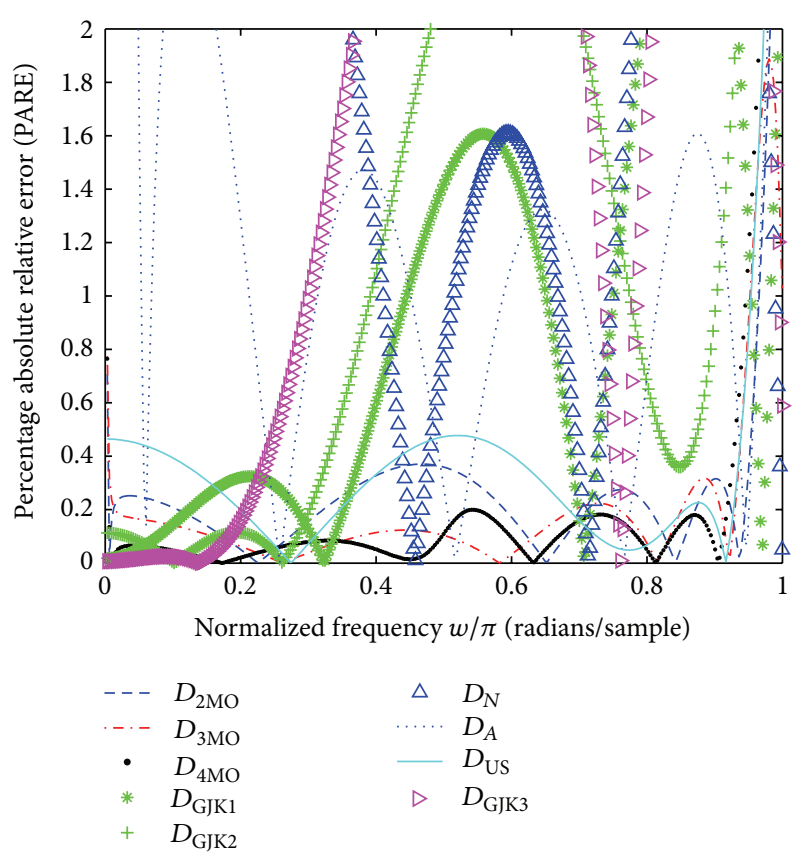

FIGURE 9: Percentage absolute relative error response of designed differentiators; $D_{2 \mathrm{MO}}(z), D_{3 \mathrm{MO}}(z)$, and $D_{4 \mathrm{MO}}(z)$, Ngo differentiator $D_{\mathrm{N}}(z)$ [6], Gupta et al. 1 differentiator $D_{\mathrm{GJK} 1}(z)$ [8], Gupta et al. 2 differentiator $D_{\mathrm{GJK} 2}(z)$ [9], Gupta et al. 3 differentiator $D_{\mathrm{GJK} 3}(z)$ [10], Al-Alaoui differentiator $D_{\mathrm{A}}(z)$ [15], and Upadhyay and Singh differentiator $D_{\mathrm{US}}(z)$ [16].

Their transfer functions are

$$
\begin{aligned}
& D_{\mathrm{N}}(z) \\
&=\left(2.7925\left(1-z^{-1}\right)\right) \\
& \quad \times\left(T_{S}(2.3658)\left(1+0.4226 z^{-1}\right)\right. \\
&\left.\quad \times\left(1-0.2605 z^{-1}+0.047 z^{-2}\right)\right)^{-1},
\end{aligned}
$$

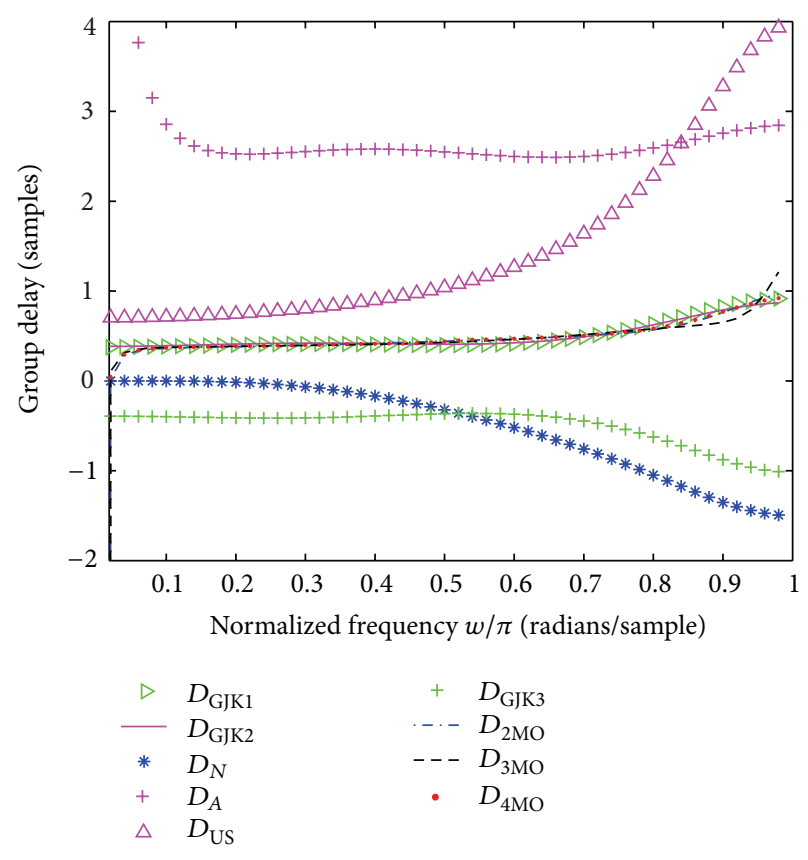

FIGURE 10: Group delay response of designed differentiators; $D_{2 \mathrm{MO}}(z), D_{3 \mathrm{MO}}(z)$, and $D_{4 \mathrm{MO}}(z)$, Ngo differentiator $D_{\mathrm{N}}(z)[6]$, Gupta et al. 1 differentiator $D_{\mathrm{GJK} 1}(z)$ [8], Gupta et al. 2 differentiator $D_{\mathrm{GJK} 2}(z)$ [9], Gupta et al. 3 differentiator $D_{\mathrm{GJK} 3}(z)$ [10], Al-Alaoui differentiator $D_{\mathrm{A}}(z)$ [15], and Upadhyay and Singh differentiator $D_{\text {US }}(z)$ [16].

$$
\begin{aligned}
& D_{\mathrm{GJK} 1}(z) \\
&=\left(1-z^{-1}\right) \\
& \quad \times\left(0.34 T_{S}(2.541)\left(1+0.3935 z^{-1}\right)\right. \\
&\left.\quad \times\left(1-0.2081 z^{-1}+0.03858 z^{-2}\right)\right)^{-1}
\end{aligned}
$$




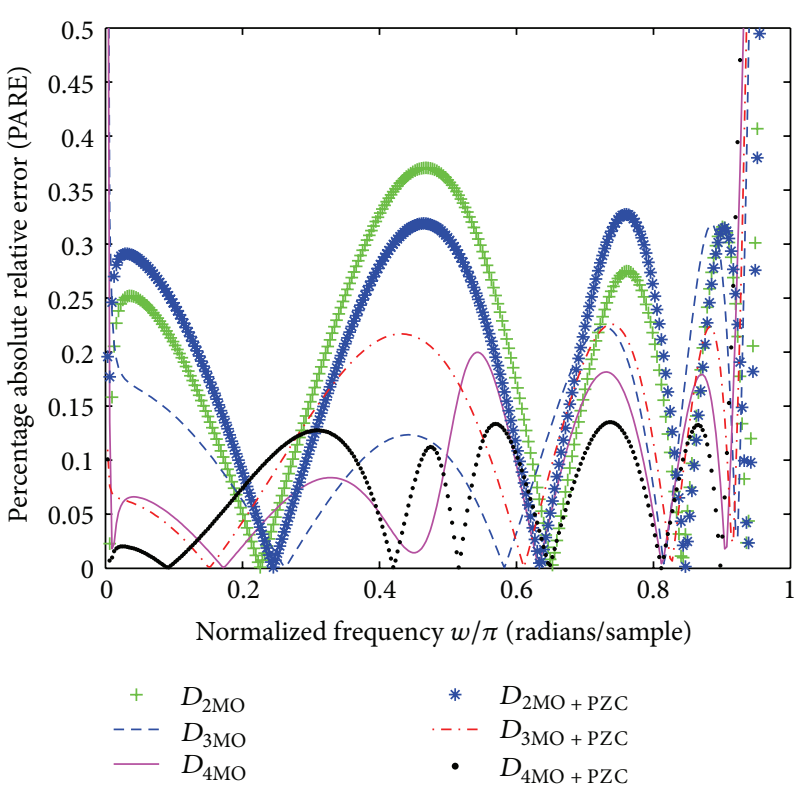

FIgURE 11: Percentage absolute relative error response of the designed differentiators; $D_{2 \mathrm{MO}}(z), D_{3 \mathrm{MO}}(z), D_{4 \mathrm{MO}}(z), D_{2 \mathrm{MO}+\mathrm{PZC}}(z)$, $D_{3 \mathrm{MO}+\mathrm{PZC}}(z)$, and $D_{4 \mathrm{MO}+\mathrm{PZC}}(z)$.

$$
\begin{aligned}
D_{\mathrm{GJK} 2}(z) & \left(1-z^{-1}\right) \\
= & \left(0.329 T_{S}(2.663)\left(1+0.3755 z^{-1}\right)\right. \\
& \left.\times\left(1-0.2079 z^{-1}+0.03864 z^{-2}\right)\right)^{-1}, \\
D_{\mathrm{GJK} 3} & (z) \\
= & \left(1-z^{-1}\right) \\
& \times\left(0.38125 T_{S}(2.285)\left(1+0.4376 z^{-1}\right)\right. \\
D_{\mathrm{A}}( & \left.\times\left(1-0.247 z^{-1}+0.04543 z^{-2}\right)\right)^{-1}, \\
= & \frac{\left(0.01903-0.02905 z^{-1}+1.123 z^{-2}-1.181 z^{-3}\right)}{T_{S}\left(1+0.1846 z^{-1}-0.001748 z^{-2}+0.03484 z^{-3}\right)}, \\
D_{\mathrm{US}}(z) & \frac{0.5805\left(1+0.99 z^{-1}-1.99 z^{-2}\right)}{T_{S}\left(1+0.681 z^{-1}+0.0628 z^{-2}\right)} .
\end{aligned}
$$

The PARE response of the designed differentiators $D_{2 \mathrm{MO}}(z)$, $D_{3 \mathrm{MO}}(z), D_{4 \mathrm{MO}}(z)$, and above mentioned existing differentiators (for $T_{S}=1$ ) is shown in Figure 9. The comparison of designed and existing differentiators on the basis of mean PARE, maximum PARE, and useful frequency range is shown in Table 5.

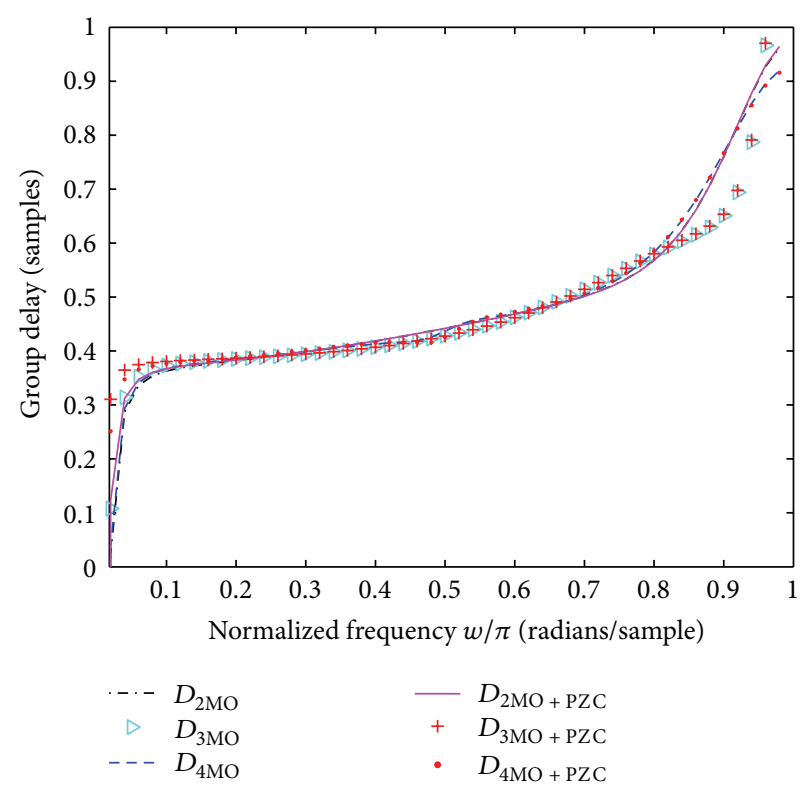

FIGURE 12: Group delay response of the designed differentiators; $D_{2 \mathrm{MO}}(z), D_{3 \mathrm{MO}}(z), D_{4 \mathrm{MO}}(z), D_{2 \mathrm{MO}+\mathrm{PZC}}(z), D_{3 \mathrm{MO}+\mathrm{PZC}}(z)$, and $D_{4 \mathrm{MO}+\mathrm{PZC}}(z)$.

The group delay response of the designed differentiators $D_{2 \mathrm{MO}}(z), D_{3 \mathrm{MO}}(z), D_{4 \mathrm{MO}}(z)$, and above mentioned existing differentiators is shown in Figure 10. It is seen that the maximum deviation from constant group delay in case of $D_{2 \mathrm{MO}}(z), D_{3 \mathrm{MO}}(z)$, and $D_{4 \mathrm{MO}}(z)$ are 0.5 samples, 0.8 samples and 0.4 samples, respectively. While, the maximum deviation from constant group delay in case of the existing differentiators Ngo [6], Gupta et al. 1 [8], Gupta et al. 2 [9], Gupta et al. 3 [10], Al-Alaoui [15], and Upadhyay and Singh [16] are 1.5 samples, 0.55 samples, 0.45 samples, 1.0 samples, 1.4 samples, and 3.3 samples, respectively.

It is verified from Figures 9 and 10 and Table 5 that the minimax optimized designed second-, third-, and fourthorder differentiators outperform all the existing differentiators over entire Nyquist frequency range.

The PARE response of the designed differentiators $D_{2 \mathrm{MO}}(z), D_{3 \mathrm{MO}}(z), D_{4 \mathrm{MO}}(z), D_{2 \mathrm{MO}+\mathrm{PZC}}(z), D_{3 \mathrm{MO}+\mathrm{PZC}}(z)$, and $D_{4 \mathrm{MO}+\mathrm{PZC}}(z)$ is shown in Figure 11. The designed differentiators $D_{2 \mathrm{MO}+\mathrm{PZC}}(z), D_{3 \mathrm{MO}+\mathrm{PZC}}(z)$, and $D_{4 \mathrm{MO}+\mathrm{PZC}}(z)$ have PARE $\leq 0.31$ over $0 \leq \omega \leq 0.95 \pi$ radians/second, $\leq 0.22$ over $0 \leq \omega \leq 0.94 \pi$ radians/second and $\leq 0.12$ over $0 \leq \omega \leq$ $0.91 \pi$ radians/second, respectively. The group delay response of the designed differentiators $D_{2 \mathrm{MO}}(z), D_{3 \mathrm{MO}}(z), D_{4 \mathrm{MO}}(z)$, $D_{2 \mathrm{MO}+\mathrm{PZC}}(z), D_{3 \mathrm{MO}+\mathrm{PZC}}(z)$, and $D_{4 \mathrm{MO}+\mathrm{PZC}}(z)$ is shown in Figure 12. It is verified from Figures 11 and 12 and Table 5 that PZC optimization improves frequency (both magnitude and phase) response of minimax optimized differentiators. In this way, a perfect family of second-, third-, and fourthorder wideband stable digital differentiators are designed with superior frequency response.

The triangular wave response of designed and existing differentiators is shown in Figures 13 and 14, respectively. It is seen that the response of designed differentiators 


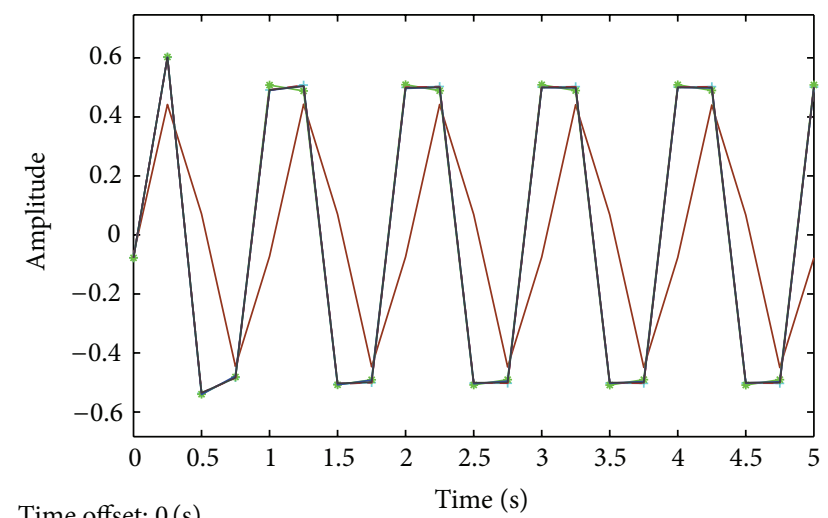

Time offset: $0(\mathrm{~s})$

$$
\begin{aligned}
& - \text { Input } \\
& --D_{2 \mathrm{MO}} \\
& -* D_{3 \mathrm{MO}} \\
& ---D_{4 \mathrm{MO}}
\end{aligned}
$$

$$
\begin{array}{ll}
--- & D_{2 \mathrm{MO}}+\mathrm{PZC} \\
- & D_{3 \mathrm{MO}+\mathrm{PZC}} \\
- & D_{4 \mathrm{MO}}+\mathrm{PZC}
\end{array}
$$

Figure 13: Triangular wave response of the designed differentiators; $D_{2 \mathrm{MO}}(z), D_{3 \mathrm{MO}}(z), D_{4 \mathrm{MO}}(z), D_{2 \mathrm{MO}+\mathrm{PZC}}(z), D_{3 \mathrm{MO}+\mathrm{PZC}}(z)$, and $D_{4 \mathrm{MO}+\mathrm{PZC}}(z)$.

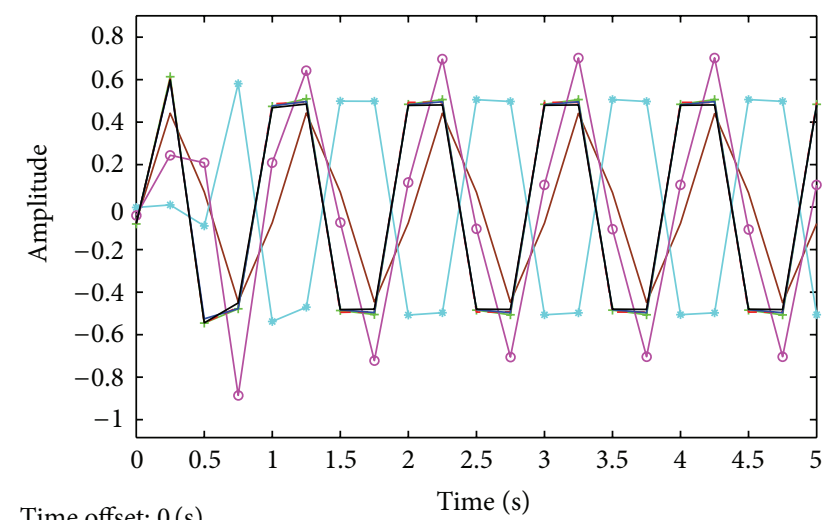

Time offset: $0(\mathrm{~s})$

$$
\begin{aligned}
& - \text { Input } \\
& -D_{N} \\
& \rightarrow-D_{A} \\
& \rightarrow D_{\text {US }}
\end{aligned}
$$$$
\begin{array}{ll}
-- & D_{\mathrm{GJK} 1} \\
- & D_{\mathrm{GJK} 2} \\
- & D_{\mathrm{GJK}}
\end{array}
$$

FIGURE 14: Triangular wave response of existing differentiators; Ngo $D_{\mathrm{N}}(z)[6]$, Gupta et al. $1 D_{\mathrm{GJK} 1}(z)$ [8], Gupta et al. $2 D_{\mathrm{GJK} 2}(z)$ [9], Gupta et al. $3 D_{\mathrm{GJK} 3}(z)$ [10], Al-Alaoui $D_{\mathrm{A}}(z)$ [15], and Upadhyay and Singh $D_{\mathrm{US}}(z)[16]$.

$D_{2 \mathrm{MO}}(z), D_{3 \mathrm{MO}}(z), D_{4 \mathrm{MO}}(z), D_{2 \mathrm{MO}+\mathrm{PZC}}(z), D_{3 \mathrm{MO}+\mathrm{PZC}}(z)$, and $D_{4 \mathrm{MO}+\mathrm{PZC}}(z)$ is exact square wave, while the response of existing differentiators is distorted square wave.

\section{Conclusion}

This paper is focused on the use of two optimization methods, namely, minimax and pole, zero, and constant (PZC). It has been proved that the efficiency of minimax optimized integrators is remarkably improved by PZC optimization. Subsequently, by modifying the transfer function of these designed integrators appropriately, new differentiators are obtained which have the same accuracy as the designed integrators. The family of designed recursive integrators and differentiators are important when excellent magnitude and linear phase response is required at the same time.

\section{References}

[1] J. L. Bihan, "Novel class of digital integrators and differentiators," Electronics Letters, vol. 29, no. 11, pp. 971-973, 1993.

[2] A. V. Oppenheim, R. W. Schafer, and J. R. Buck, Discrete-Time Signal Processing, Prentice-Hall, 1999.

[3] S. K. Mitra, Digital Signal Processing a Computer Based Approach, Tata McGraw Hill, 2005.

[4] A. M. Schneider, J. T. Kaneshige, and F. D. Groutage, "Higher order s-to-z mapping functions and their application in digitizing continuous-time filters," Proceedings of the IEEE, vol. 79, no. 11, pp. 1661-1674, 1991.

[5] M. A. Al-Alaoui, "Novel digital integrator and differentiator," Electronics Letters, vol. 29, no. 4, pp. 376-378, 1993.

[6] N. Q. Ngo, "A new approach for the design of wideband digital integrator and differentiator," IEEE Transactions on Circuits and Systems II: Express Briefs, vol. 53, no. 9, pp. 936-940, 2006.

[7] C. C. Tseng and S. L. Lee, "Digital IIR integrator design using Richardson extrapolation and fractional delay," IEEE Transactions on Circuits and Systems Part I: Regular Papers, vol. 55, no. 8, pp. 2300-2309, 2008.

[8] M. Gupta, M. Jain, and B. Kumar, "Novel class of stable wideband recursive digital integrators and differentiators," IET Signal Processing, vol. 4, no. 5, pp. 560-566, 2010.

[9] M. Gupta, M. Jain, and B. Kumar, "Recursive wideband digital integrator and differentiator," International Journal of Circuit Theory and Applications, vol. 39, no. 7, pp. 775-782, 2011.

[10] M. Gupta, M. Jain, and B. Kumar, "Wideband digital integrator and differentiator," IETE Journal of Research, vol. 58, no. 2, pp. 166-170, 2012.

[11] M. Gupta, M. Jain, and B. Kumar, "Wideband digital integrator," in Proceedings of the IEEE International Conference on Multimedia, Signal Processing and Communication Technologies (IMPACT '09), pp. 107-109, Aligarh, India, 2009.

[12] M. Gupta, M. Jain, and B. Kumar, "Design of a novel digital integrator," in Proceedings of the IEEE International Conference on Aerospace Electronics, Communications and Instrumentation (ASECI '10), pp. 191-193, Andhra Pradesh, India, 2010.

[13] M. Gupta, M. Jain, and N. Jain, "A new fractional order recursive digital integrator using continued fraction expansion," in Proceedings of the IEEE International Conference on Power Electronics (IICPE '10), pp. 1-3, Delhi, India, 2010.

[14] N. Papamarkos and C. Chamzas, "A new approach for the design of digital integrators," IEEE Transactions on Circuits System Part I: Fundamental Theory and Applications, vol. 43, no. 9, pp. 785-791, 1996.

[15] M. A. Al-Alaoui, "Class of digital integrators and differentiators," IET Signal Processing, vol. 5, no. 2, pp. 251-260, 2011.

[16] D. K. Upadhyay and R. K. Singh, "Recursive wideband digital differentiator and integrator," Electronics Letters, vol. 47, no. 11, pp. 647-648, 2011.

[17] A. Jiang and H. K. Kwan, "Minimax design of IIR digital filters using SDP relaxation technique," IEEE Transactions on Circuits and Systems I: Regular Papers, vol. 57, no. 2, pp. 378-390, 2010. 
[18] X. P. Lai and Z. P. Lin, "Minimax design of IIR digital filters using a sequential constrained least-squares method," IEEE Transactions on Signal Processing, vol. 58, no. 7, pp. 3901-3906, 2010.

[19] C. C. Hsu, W. Y. Wang, and C. Y. Yu, "Genetic algorithm-derived digital integrators and their applications in discretization of continuous systems," in Proceedings of the CEC Congress on Evolutionary Computation, pp. 443-448, Honolulu, Hawaii, USA, 2002.

[20] J. M. Renders and S. P. Flasse, "Hybrid methods using genetic algorithms for global optimization," IEEE Transactions on Systems, Man, and Cybernetics, Part B, vol. 26, no. 2, pp. 243258, 1996.

[21] Y. Wu, X. Z. Gao, X. L. Huang, and K. Zenger, "A hybrid optimization method of particle swarm optimization and cultural algorithm," in Proceedings of the 6th International Conference on Natural Computation (ICNC '10), pp. 2515-2519, August 2010.

[22] M. R. Stojic and R. M. Stojic, "Pole-zero sensitivities of a digital filter due to parameter quantization," International Journal of Circuit Theory and Applications, vol. 5, no. 3, pp. 299-304, 1977.

[23] A. Hiltgen and G. S. Moschytz, "A pole-zero pairing strategy for minimum-gain-sensitivity $n$ th-order biquadratic filter cascades," International Journal of Circuit Theory and Applications, vol. 18, no. 2, pp. 145-163, 1990.

[24] M. A. Al-Alaoui, "Novel approach to designing digital differentiators," Electronics Letters, vol. 28, no. 15, pp. 1376-1378, 1992. 

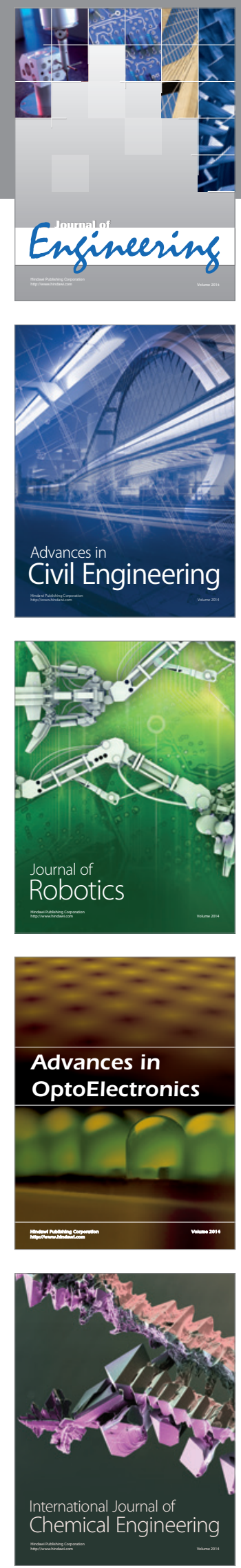

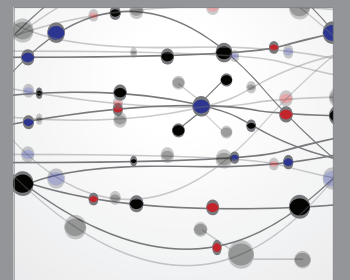

The Scientific World Journal
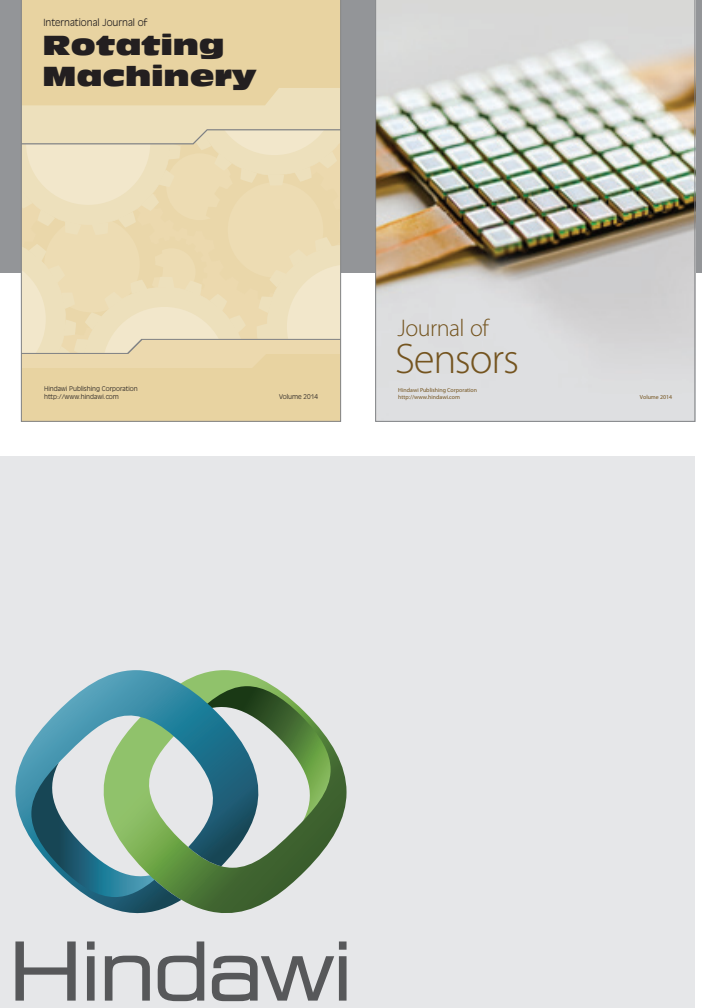

Submit your manuscripts at http://www.hindawi.com
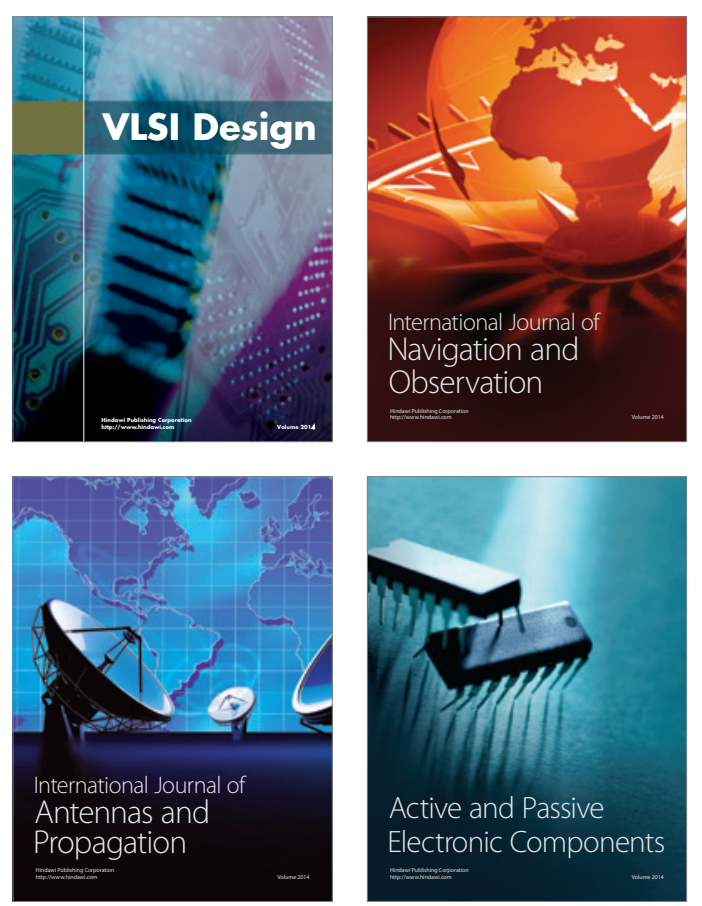
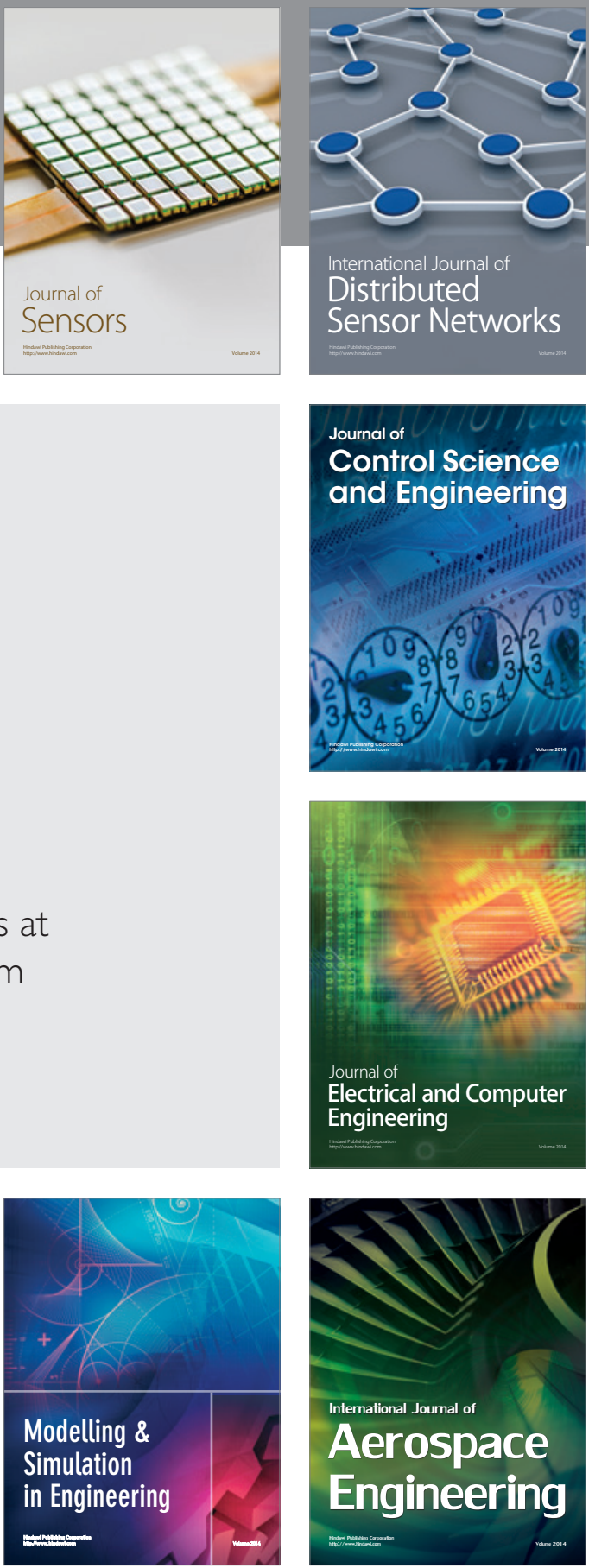

Journal of

Control Science

and Engineering
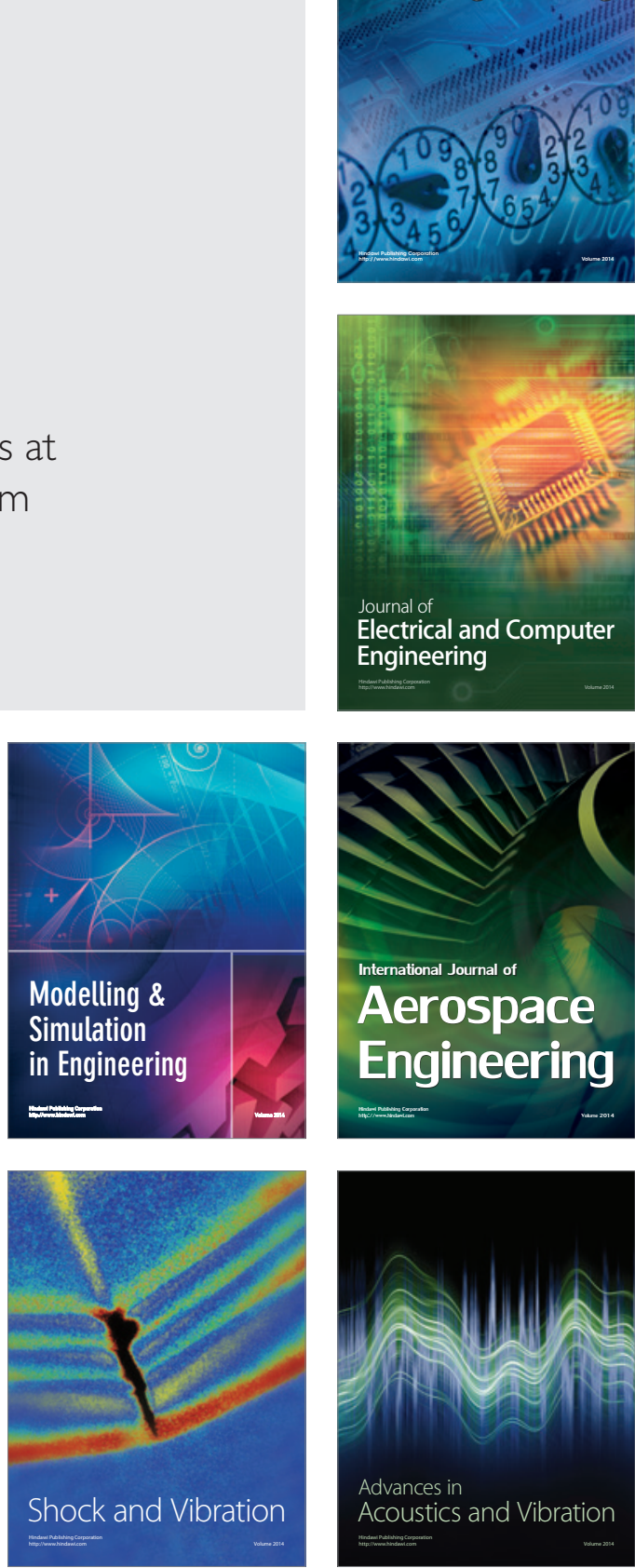